Comment. Math. Helv. 75 (2000) 608-643

$0010-2571 / 00 / 040608-36 \$ 1.50+0.20 / 0$
(C) 2000 Birkhäuser Verlag, Basel

Commentarii Mathematici Helvetici

\title{
Strong approximation for Zariski dense subgroups over arbitrary global fields
}

\author{
Richard Pink
}

\begin{abstract}
Consider a finitely generated Zariski dense subgroup $\Gamma$ of a connected simple algebraic group $G$ over a global field $F$. An important aspect of strong approximation is the question of whether the closure of $\Gamma$ in the group of points of $G$ with coefficients in a ring of partial adeles is open. We prove an essentially optimal result in this direction, based on the condition that $\Gamma$ is not discrete in that ambient group. There are no restrictions on the characteristic of $F$ or the type of $G$, and simultaneous approximation in finitely many algebraic groups is also studied. Classification of finite simple groups is not used.
\end{abstract}

Mathematics Subject Classification (2000). Primary 20G35; Secondary 11F06, 11R58, 20G30, 20H20.

\section{Introduction}

Consider a connected semisimple linear algebraic group $G$ over a global field $F$ and a finitely generated Zariski dense subgroup $\Gamma \subset G(F)$. For any finite set $S$ of places of $F$ we let $\mathbb{A}_{F}^{S}$ denote the ring of adeles of $F$ outside $S$. In this article we study the closure of $\Gamma$ in $G\left(\mathbb{A}_{F}^{S}\right)$. We are interested in sufficient conditions for such a closure to be open, in which case we speak of strong approximation. For a general survey of approximation in algebraic groups see Platonov and Rapinchuk [17] Ch. 7.

It is best to reformulate the problem in terms of absolutely simple groups. At the same time, it can be generalized. Consider finitely many connected absolutely simple linear algebraic groups $G_{i}$ over global fields $F_{i}$, and a finitely generated subgroup $\Gamma \subset \prod_{i=1}^{m} G_{i}\left(F_{i}\right)$ whose image in each factor is Zariski dense. No relations between the $F_{i}$ need be assumed; in particular, even their characteristics may differ.

It is well-known that strong approximation fails in non-simply connected groups, even when $\Gamma$ is an arithmetic subgroup. To deal with this difficulty we assume that every $G_{i}$ is adjoint and study approximation in the universal coverings $\tilde{G}_{i} \rightarrow G_{i}$. The commutator morphism of $\tilde{G}_{i}$ factors through a unique morphism

$$
[,]^{\sim}: G_{i} \times G_{i} \longrightarrow \tilde{G}_{i}
$$


The generalized commutator group of $\Gamma$ is the subgroup $\Gamma^{\prime} \subset \prod_{i=1}^{m} \tilde{G}_{i}\left(F_{i}\right)$ generated by the generalized commutators $[\Gamma, \Gamma]^{\sim}$. All we can hope for is that the closure of $\Gamma^{\prime}$ in $\prod_{i=1}^{m} \tilde{G}_{i}\left(\mathbb{A}_{F_{i}}^{S_{i}}\right)$ is open.

Let $G$ denote the group scheme over $F:=\bigoplus_{i=1}^{m} F_{i}$ with fibers $G_{i}$. Then we have $G(F)=\prod_{i=1}^{m} G_{i}\left(F_{i}\right)$. It may happen that the coefficients of $\Gamma$ lie in a proper subring of $F$. A similar problem, relevant only in small positive characteristic, is that $\Gamma$ may be contained in the image of a non-central isogeny. In these cases it is best to study approximation over the subring, resp. after pullback via the isogeny. A coherent treatment is given as follows.

Consider a subring $E \subset F$ which is itself a finite direct sum of global fields, such that $F$ is of finite type as $E$-module. Consider a group scheme $H$ over $E$ with connected adjoint fibers, and an isogeny $\varphi: H \times_{E} F \longrightarrow G$, such that $\Gamma \subset \varphi(H(E))$. The triple $(E, H, \varphi)$ is then called a weak quasi-model of $(F, G, \Gamma)$. We suppose that the derivative of $\varphi$ vanishes nowhere; such a triple is called a quasi-model of $(F, G, \Gamma)$.

When $\varphi$ is an isomorphism, we can view $H$ as a model of $G$ over $E$ in the usual sense. By the classification of semisimple groups, it must be an isomorphism unless some $G_{i}$ possesses roots of different lengths whose square length ratio is equal to $\operatorname{char}\left(F_{i}\right)$. In the remaining cases, which can happen only in characteristics 2 and 3 , one cannot avoid non-standard isogenies.

For any quasi-model, the fact that $H$ has adjoint fibers implies that the isogeny $\varphi$ is totally inseparable. Therefore the induced map $H(E) \rightarrow G(F)$ is injective, and we may identify $\Gamma$ with its inverse image in $H(E)$. We can then replace the triple $(F, G, \Gamma)$ by the triple $(E, H, \Gamma)$, which satisfies the same conditions as the former. It is known that this process cannot be iterated indefinitely, unless $E=F$ and $\varphi$ is an isomorphism. More precisely, after passing to an essentially unique minimal quasi-model the triple $(F, G, \Gamma)$ satisfies the following property, which we assume from now on (cf. Section 3):

Assumption 0.1. For every weak quasi-model $(E, H, \varphi)$ of $(F, G, \Gamma)$, we have $E=F$ and $\varphi$ is an isomorphism.

We first state our main result for approximation at compact places. For every $1 \leq i \leq m$ let $S_{i, \Gamma}$ denote the set of places $v$ of $F_{i}$ for which the image of $\Gamma$ in $G_{i}\left(F_{i, v}\right)$ does not lie in a compact subgroup. It is easy to see that $S_{i, \Gamma}$ is finite: see Corollary 7.2.

Theorem 0.2. The closure of $\Gamma^{\prime}$ in $\prod_{i=1}^{m} \tilde{G}_{i}\left(\mathbb{A}_{F_{i}}^{S_{i, \Gamma}}\right)$ is open.

The following more general result gives a necessary and sufficient condition for strong approximation outside an arbitrary finite set of places. For every $1 \leq i \leq m$ consider a finite set $S_{i}$ of places of $F_{i}$. 
Theorem 0.3. The following assertions are equivalent:

(a) For any non-empty subset $I \subset\{1, \ldots, m\}$, the image of $\Gamma$ in $\prod_{i \in I} G_{i}\left(\mathbb{A}_{F_{i}}^{S_{i}}\right)$ is not discrete.

(b) The closure of $\Gamma^{\prime}$ in $\prod_{i=1}^{m} \tilde{G}_{i}\left(\mathbb{A}_{F_{i}}^{S_{i}}\right)$ is open.

When $\Gamma$ is an arithmetic subgroup such results are well-known: see for instance Kneser [14], Prasad [18], Platonov and Rapinchuk [17]. For arbitrary finitely generated Zariski dense subgroups Theorem 0.2 was proved by Weisfeiler ([21] Thms. 9.1.1 and 10.1) over the adeles outside an indeterminate finite set of places, assuming that $G$ possesses no non-standard isogenies. He also proved a generalization to fields of arbitrary transcendence degree. Our treatment here follows the lines laid out in [21] but incorporates several improvements.

The inclusion of the non-standard cases was made possible by a detailed analysis of their idiosyncracies, which was suggested in [21] Sect. 12 and begun by the author in [16].

The improvement in the choice of the discarded places has much larger scope. Namely, even the weak approximation property at a previously given finite set of places where $\Gamma$ is relatively compact, that is, the openness of the closure in a finite product of $\tilde{G}_{i}\left(F_{i, v}\right)$, is entirely non-trivial in positive characteristic. It was proved in [16] with - originally - a different application in mind, and found to be useful in the present context.

Thirdly, we are able to avoid recourse to the classification of finite simple groups, using the qualitative characterization of finite subgroups of linear algebraic groups by Larsen and the author [15] instead.

In Section 1 we review a number of less well-known properties of simple algebraic groups over arbitrary fields. Section 2 deals with groups over local fields. In Sections 3-4 we restate several concepts and results from [16]. After these preparations we study weak approximation at any given finite set of places in Sections 5-6. The complementary problem, that is, the behavior of $\Gamma$ at all but a large finite set of places, is the subject of Sections 7-12. These results are combined in Sections $13-14$, where Theorems 0.2 and 0.3 are proved. The reader interested only in Theorem 0.2 may skip Sections 2, 6, and 14 .

The author wishes to thank Michael Larsen for helpful remarks on earlier versions of this paper.

\section{Isogenies and the adjoint representation}

We begin by recalling some aspects of simple algebraic groups which were explained in [16] Sect. 1. Consider a connected adjoint absolutely simple linear algebraic group $G$ over a field $F$. We call $G$ non-standard if it possesses roots of different lengths whose square length ratio is equal to $\operatorname{char}(F)$. Otherwise $G$ is called standard. Non-standard groups exist only in characteristics 2 and 3. 
Isogenies: If $p:=\operatorname{char}(F)$ is positive and $\sigma: F \rightarrow F$ denotes the basic Frobenius homomorphism $x \mapsto x^{p}$, there are the natural Frobenius isogenies Frob $p_{p^{r}}: G \rightarrow$ $\left(\sigma^{r}\right)^{*} G$. In the standard case every isogeny between $G$ and another connected adjoint simple group is a composite of a Frobenius isogeny with an isomorphism. In particular, it is an isomorphism if and only if its derivative is non-zero.

In the non-standard case there exists a basic non-standard isogeny to another adjoint simple group $\varphi_{0}: G \rightarrow G^{\sharp}$, whose derivative is non-zero but which is not an isomorphism. In this case every isogeny between $G$ and another connected adjoint simple group is either a composite of a Frobenius isogeny with an isomorphism, or a composite of $\varphi_{0}$ with a Frobenius isogeny and an isomorphism. In particular, its derivative is non-zero if and only if it is either an isomorphism or a basic non-standard isogeny. The complementary group $G^{\sharp}$ is again non-standard, and the composite of the two respective basic non-standard isogenies differs from the Frobenius isogeny Frob $_{p}$ by an isomorphism.

Giving a model $G_{0}$ of $G$ over a finite subfield $\mathbb{F}_{p^{r}} \subset F$ is equivalent to giving an isomorphism $\left(\sigma^{r}\right)^{*} G \cong G$. Its composite with Frob $p_{p^{r}}$ is the associated standard Frobenius map. An arbitrary isogeny $\Phi: G \rightarrow G$ is called a Frobenius map if and only if some positive power is a standard Frobenius map. Suppose that $F$ is algebraically closed. Then the group of fixed points $G^{\Phi}$ is called a finite group of Lie type. If $\Phi$ is standard, we have $G^{\Phi}=G_{0}\left(\mathbb{F}_{p^{r}}\right)$; otherwise $G^{\Phi}$ is a Suzuki or Ree group.

Adjoint representation: In the standard case the Lie algebra of $G$ possesses a unique irreducible subquotient on which $G$ acts non-trivially. We denote the representation on it by $\alpha$.

In the non-standard case there are precisely two simple subquotients with nontrivial $G$-action, one of which contains copies of all long root spaces, the other of all short root spaces. The corresponding representations of $G$ are denoted $\alpha_{\ell}$ and $\alpha_{s}$. The derivative of the basic non-standard isogeny $\varphi_{0}$ induces an isomorphism $\alpha_{\ell} \cong$ $\alpha_{s} \circ \varphi_{0}$. We sometimes view $\alpha_{\ell} \oplus \alpha_{s}$ as a representation over $F \oplus F$ and denote it also by $\alpha$.

Let $\pi: \tilde{G} \rightarrow G$ denote the universal covering. We abbreviate $\tilde{L}_{G}:=$ Lie $\tilde{G}$ and $L_{G}:=\operatorname{Lie} G$. The fine structure of these representations was determined by Hiss [12] and Hogeweij [13] (see also [16] Prop. 1.11). We briefly describe some of those facts which are needed below.

The kernel of $d \pi: \tilde{L}_{G} \rightarrow L_{G}$ is the subspace of $G$-invariants of $\tilde{L}_{G}$, the cokernel the space of $G$-coinvariants of $L_{G}$. Their common dimension is 0 unless $p$ divides the index of the root lattice in the weight lattice. In that case it is 1 , except if $p=2$ and the root system has type $D_{n}$ with $n \geq 4$ even, where it is 2 .

Let $\tilde{M}_{G}$ denote the representation space of $\alpha$ in the standard case, resp. of $\alpha_{\ell}$ in the non-standard case. It is known that $\tilde{L}_{G}$ possesses no proper invariant subspace with non-trivial image in $\tilde{M}_{G}$. Thus $\tilde{M}_{G}$ is the unique simple quotient of $\tilde{L}_{G}$. Let $M_{G}:=L_{G}$ in the standard case, resp. $M_{G}:=\operatorname{im}\left(d \varphi_{0}\right)$ in the non- 
standard case. Note that in the non-standard case the representation of $G$ on $M_{G}$ factors through $\varphi_{0}$. In any case $M_{G}$ is the unique smallest quotient of $L_{G}$ into which $\tilde{M}_{G}$ injects. We give names to the respective kernels, as in the commutative diagram with exact rows

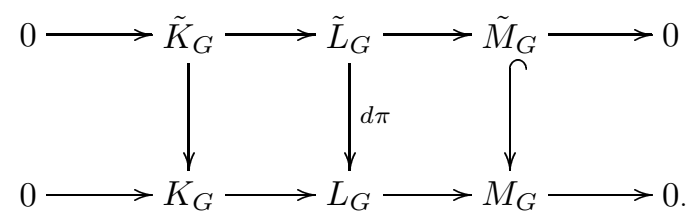

The natural map Coker $(d \pi) \longrightarrow M_{G} / \tilde{M}_{G}$ is an isomorphism unless $p=2$ and $G$ has type $B_{n}$ with $n$ odd. In particular, we have $\operatorname{dim}\left(M_{G} / \tilde{M}_{G}\right)=1$ whenever $p=2$ and $G$ has type $C_{n}$ for any $n \geq 1$.

Lie bracket: The commutator map of $\tilde{G}$ factors through a morphism

$$
[,]^{\sim}: G \times G \longrightarrow \tilde{G}
$$

Its total derivative at the identity element defines a generalized Lie bracket

$$
[,]^{\sim}: L_{G} \times L_{G} \longrightarrow \tilde{L}_{G}
$$

denoted by the same symbol. Its composite with the map $d \pi: \tilde{L}_{G} \rightarrow L_{G}$ is the usual Lie bracket [, ] on $\tilde{L}_{G}$, resp. on $L_{G}$. The induced pairing $L_{G} \times \tilde{L}_{G} \rightarrow \tilde{L}_{G}$ is also denoted $[,]^{\sim}$. The images of these pairings generate the following subspaces.

\section{Proposition 1.2.}

(a) We have $\left[\tilde{L}_{G}, \tilde{L}_{G}\right]=\tilde{L}_{G}$ unless $\operatorname{char}(F)=2$ and the root system of $G$ has type $C_{n}$ for some $n \geq 1$. In that case we have $\left[\tilde{L}_{G}, \tilde{L}_{G}\right] \subset \tilde{K}_{G}$.

(b) In any case we have $\left[L_{G}, \tilde{L}_{G}\right]^{\sim}=\tilde{L}_{G}$.

Proof. Like [16] Props. 1.11 and 1.12, this can be proved by explicit calculation using the root space decomposition and the well-known structure constants of Chevalley groups. It can also be deduced directly from [16] Prop. 1.12. This result shows that the representation of $\tilde{G}$ on $\tilde{M}_{G}$ factors through Frob ${ }_{p}: \tilde{G} \rightarrow \sigma^{*} \tilde{G}$ if and only if $p:=\operatorname{char}(F)=2$ and $G$ has type $C_{n}$ with $n \geq 1$. Taking derivatives we find that the image of $\left[\tilde{L}_{G}, \tilde{L}_{G}\right]$ in $\tilde{M}_{G}$ is zero exactly in this special case. Otherwise $\left[\tilde{L}_{G}, \tilde{L}_{G}\right]$ is an invariant subspace of $\tilde{L}_{G}$ with non-trivial image in $\tilde{M}_{G}$. It is therefore equal to $\tilde{L}_{G}$, which proves (a). Part (b) is proved in the same way, using the fact that the representation on $\tilde{M}_{G}$ never factors through Frob $: G \rightarrow \sigma^{*} G$. 


\section{Simple algebraic groups over local fields}

In this brief section we assume that $F$ is a local field, i.e., a locally compact field with non-trivial valuation. As in Section 1, we consider a connected adjoint absolutely simple linear algebraic group $G$ over $F$ and let $\pi: \tilde{G} \rightarrow G$ denote its universal covering.

Proposition 2.1. The factor group $G(F) / \pi(\tilde{G}(F))$ is abelian of finite exponent.

Proof. The factor group is contained in the cohomology group $H^{1}(F, \mu)$, where $\mu$ denotes the scheme-theoretic kernel of $\pi$ (see [10]). Let $m$ denote the index of the root lattice in the weight lattice for $G$. Then $\mu$ is commutative and annihilated by $m$. The cohomology group retains these properties, and so does any subgroup, as desired.

Theorem 2.2. Any open subgroup of $\tilde{G}(F)$ which is normalized by an unbounded subgroup of $G(F)$ is equal to $\tilde{G}(F)$.

Proof. If $F$ is archimedean, the group $\tilde{G}(F)$ is connected by [3] Cor. 4.7. Therefore any open subgroup is equal to $\tilde{G}(F)$, as desired. Assume that $F$ is non-archimedean and let $\mathscr{O}$ denote the ring of integers in $F$. Consider an open subgroup $\Delta \subset \tilde{G}(F)$ which is normalized by an unbounded subgroup $\Gamma \subset G(F)$.

Lemma 2.3. The group $\Delta$ is unbounded.

Proof. Consider a non-trivial absolutely irreducible representation $\rho: G \rightarrow \mathrm{GL}_{n, F}$, say occurring in the adjoint representation of $G$. Suppose that $\Delta$ is bounded. Then the image $\rho \circ \pi(\Delta)$ is bounded and so is the $\mathscr{O}$-subalgebra $\mathscr{A}$ generated by it. As $\Delta$ is Zariski dense in $\tilde{G}$, its action on $F^{n}$ is also absolutely irreducible. Thus Burnside's theorem implies that $\mathscr{A}$ is an $\mathscr{O}$-lattice of maximal rank in the space of $n \times n$-matrices over $F$. By construction $\Gamma$ stabilizes this lattice under the conjugation representation $\rho \otimes \rho^{\vee}$. Therefore the image of $\Gamma$ under the corresponding projective representation $\bar{\rho}: G \rightarrow \mathrm{PGL}_{n, F}$ is bounded. Since $G \rightarrow \bar{\rho}(G)$ is an isogeny, it follows that $\Gamma$ itself is bounded, contrary to the assumption.

The existence of an unbounded subgroup implies that $\tilde{G}$ is isotropic ([19]). Therefore it satisfies the Kneser-Tits conjecture ([17] Thm. 7.6), so $\tilde{G}(F)$ is generated by the $F$-valued points of the unipotent radicals of all parabolic subgroups. A theorem of Tits ([19]) now shows that every proper open subgroup of $\tilde{G}(F)$ is bounded. Thus we must have $\Delta=\tilde{G}(F)$, as desired. 


\section{Quasi-models}

Now we recall the concept and some properties of quasi-models from [16] Sect. 3. We give the definitions in both the global and local cases, in order to set up the passage from one to the other.

Consider a commutative semisimple ring $F$, that is, a finite direct sum of fields $\bigoplus_{i=1}^{m} F_{i}$. No relation between the $F_{i}$ is assumed; even their characteristics may differ. Let $G$ be a linear algebraic group scheme over $F$ whose fibers $G_{i}$ over $F_{i}$ are connected adjoint and absolutely simple. Consider a subgroup $\Gamma \subset G(F) \cong$ $\prod_{i=1}^{m} G_{i}\left(F_{i}\right)$ whose image in each $G_{i}\left(F_{i}\right)$ is Zariski dense. We make one of the following assumptions.

\section{Assumption 3.1.}

(a) Global case: Each $F_{i}$ is a global field, i.e., a finite extension either of $\mathbb{Q}$ or of $\mathbb{F}_{p}(t)$ for some prime $p$.

(b) Local case: Each $F_{i}$ is a locally compact field with non-trivial valuation.

In [16] we had also imposed certain conditions on $\Gamma$ : see 3.6 below. We depart from this in the following definitions, because the greater generality helps in studying approximation at non-compact places.

Definition 3.2. A weak quasi-model of $(F, G, \Gamma)$ is a triple $(E, H, \varphi)$ where

(a) $E$ is a semisimple subring of $F$ such that $F$ is of finite type as E-module, and which is closed in the local case,

(b) $H$ is a fiberwise absolutely simple adjoint group over $E$, and

(c) $\varphi$ is an isogeny $H \times_{E} F \longrightarrow G$, such that

(d) $\Gamma$ is contained in the subgroup $\varphi(H(E)) \subset G(F)$.

Here, as elsewhere in this paper, we abbreviate $H \times_{E} F:=H \times_{\mathbf{S p e c}} E \operatorname{Spec} F$.

Definition 3.3. A quasi-model of $(F, G, \Gamma)$ is a weak quasi-model for which the derivative of $\varphi$ vanishes nowhere.

In any weak quasi-model the fact that $H$ is adjoint implies that $\varphi$ is totally inseparable. Therefore the induced map $H(E) \rightarrow G(F)$ is injective, so that we may identify $\Gamma$ with its pre-image in $H(E)$. Note that $\Gamma$ is automatically fiberwise Zariski dense in $H$. It follows that the triple $(E, H, \Gamma)$ satisfies the same assumptions as $(F, G, \Gamma)$.

Definition 3.4. The triple $(F, G, \Gamma)$ is called minimal if and only if, for every weak quasi-model $(E, H, \varphi)$ of $(F, G, \Gamma)$, we have $E=F$ and $\varphi$ is an isomorphism.

Definition 3.5. A (weak) quasi-model $(E, H, \varphi)$ of $(F, G, \Gamma)$ is called minimal if 
and only if $(E, H, \Gamma)$ is minimal in its own right.

The following condition was assumed throughout the paper [16], although it is actually unnecessary for some of those results:

\section{Condition 3.6.}

(a) Global case: $\Gamma$ is finitely generated.

(b) Local case: $\Gamma$ is compact.

We recall [16] Thm. 3.6:

Theorem 3.7. If $\Gamma$ satisfies Condition 3.6, then:

(a) Every $(F, G, \Gamma)$ has a minimal quasi-model $(E, H, \varphi)$.

(b) The subring $E$ in (a) is unique, and $H$ and $\varphi$ are determined up to unique isomorphism.

We now list a few useful reduction properties. The first concerns projection to a direct summand and will often allow reduction to the field case. It was proved in [16] Prop. 3.9 without reference to Condition 3.6 at all:

Proposition 3.8. Consider any subset $I \subset\{1, \ldots, m\}$. Set $F_{I}:=\bigoplus_{i \in I} F_{i}$ and $G_{I}:=\coprod_{i \in I} G_{i}$, and let $\Gamma_{I}$ denote the image of $\Gamma$ in $G_{I}\left(F_{I}\right)=\prod_{i \in I} G_{i}\left(F_{i}\right)$. If $(F, G, \Gamma)$ is minimal, then $\left(F_{I}, G_{I}, \Gamma_{I}\right)$ is minimal.

The other two concern the passage from $\Gamma$ to a subgroup. Note first that any subgroup of finite index is still fiberwise Zariski dense.

Proposition 3.9. Assume that $\Gamma$ satisfies Condition 3.6 and that $(F, G, \Gamma)$ is minimal. Then for any subgroup $\Delta \subset \Gamma$ of finite index, closed in the local case, the triple $(F, G, \Delta)$ is minimal.

Proof. Let $\Delta_{1}$ be the intersection of all $\Gamma$-conjugates of $\Delta$. Then $\left(F, G, \Delta_{1}\right)$ is minimal by [16] Cor. 3.8. As any quasi-model $(E, H, \varphi)$ of $(F, G, \Delta)$ is also a quasi-model of $\left(F, G, \Delta_{1}\right)$, we deduce $E=F$ and that $\varphi$ is an isomorphism. Thus $(F, G, \Delta)$ is minimal, as desired.

Recall that a subgroup $\Delta \subset \Gamma$ is commensurated by $\Gamma$ if, for every $\gamma \in \Gamma$, the intersection $\Delta \cap \gamma \Delta \gamma^{-1}$ has finite index in both $\Delta$ and $\gamma \Delta \gamma^{-1}$.

Proposition 3.10. Consider a subgroup $\Delta \subset \Gamma$, which is commensurated by $\Gamma$ and whose image in each $G_{i}\left(F_{i}\right)$ is Zariski dense. Assume that $\Delta$ (sic!) satisfies Condition 3.6. If $(F, G, \Gamma)$ is minimal, then $(F, G, \Delta)$ is minimal. 
Proof. By Theorem 3.7 (a) we may choose a minimal quasi-model $(E, H, \varphi)$ of $(F, G, \Delta)$. Let $\operatorname{int}(\gamma)$ denote the inner automorphism of $G$ corresponding to any given element $\gamma \in \Gamma$. Then $(E, H, \operatorname{int}(\gamma) \circ \varphi)$ is a minimal quasi-model of $\left(F, G, \gamma \Delta \gamma^{-1}\right)$. By Proposition 3.9 the minimality of both triples is preserved on passing to the subgroup of finite index $\Delta \cap \gamma \Delta \gamma^{-1}$. In other words, both $(E, H, \varphi)$ and $(E, H, \operatorname{int}(\gamma) \circ \varphi)$ are minimal quasi-models of $\left(F, G, \Delta \cap \gamma \Delta \gamma^{-1}\right)$. Therefore, by the uniqueness in Theorem 3.7 (b), there exists an automorphism $\iota$ of $H$ with $\operatorname{int}(\gamma) \circ \varphi=\varphi \circ \iota$. Since $\varphi$ induces an isomorphism between the groups of outer automorphisms of $H$ and $G$, we find that $\iota$ is an inner automorphism. As $H$ is adjoint, it follows that $\iota=\operatorname{int}(\delta)$ for some $\delta \in H(E)$. This proves $\gamma=\varphi(\delta) \in \varphi(H(E))$; hence $(E, H, \varphi)$ is a quasi-model of $(F, G, \Gamma)$. By the minimality assumption, we deduce $E=F$ and that $\varphi$ is an isomorphism. Thus $(F, G, \Delta)$ is minimal, as desired.

\section{Group rings and traces}

From here until the end of the paper we assume that $F$ is global and $\Gamma$ is finitely generated. As explained in the introduction, we pass to a minimal quasi-model using Theorem 3.7, and thus assume that $(F, G, \Gamma)$ is minimal.

We now collect some facts from [16] Sects. 3-4 concerning the action of $\Gamma$ on certain parts of the Lie algebra of $G$. We begin with the conceptually simpler results on traces. Let $(F, G, \Gamma)$ be as in the preceding section, and set

$$
F^{\prime}:=\bigoplus_{i=1}^{m} \begin{cases}F_{i} & \text { if } G_{i} \text { is standard, } \\ F_{i} \oplus F_{i} & \text { if } G_{i} \text { is non-standard. }\end{cases}
$$

Combining the representations $\alpha$ defined in Section 1 for all $G_{i}$, we obtain a representation of $G$ over $F^{\prime}$ which is fiberwise absolutely irreducible. Let $\mathscr{O}_{\operatorname{Tr}(\alpha)} \subset$ $F^{\prime}$ denote the subring generated by the set of traces $\operatorname{Tr} \alpha(\Gamma)$, and $E_{\alpha} \subset F^{\prime}$ its total ring of quotients. The minimality of $(F, G, \Gamma)$ and [16] Thm. 2.3 (b), Prop. 3.10, and Prop. 3.14 imply:

Proposition 4.2. We have

$$
E_{\alpha}=\bigoplus_{i=1}^{m} \begin{cases}E_{i} & \text { if } G_{i} \text { is standard, } \\ E_{i, \ell} \oplus E_{i, s} & \text { if } G_{i} \text { is non-standard, }\end{cases}
$$

where $E_{i}$, resp. $E_{i, \ell}$ and $E_{i, s}$, is a subfield of $F_{i}$. Moreover, this subfield is equal to $F_{i}$ except, possibly, in the cases:

(a) $\operatorname{char}\left(F_{i}\right)=2$ and the root system of $G_{i}$ has type $A_{1}$. Here $E_{i}$ is equal to $F_{i}$ or to $\left\{x^{2} \mid x \in F_{i}\right\}$.

(b) $\operatorname{char}\left(F_{i}\right)=2$ and the root system of $G_{i}$ has type $C_{n}$ for some $n \geq 2$. Here $E_{i, s}=F_{i}$, and $E_{i, \ell}$ is equal to $F_{i}$ or to $\left\{x^{2} \mid x \in F_{i}\right\}$. 
For finer information we turn to the group ring. For every representation $\rho$ of $\Gamma$ on an additive group $V$ we let $J(V) \subset \operatorname{End}(V)$ denote the image of the augmentation ideal of the group ring $\mathbb{Z}[\Gamma]$. In other words, this is the additive subgroup generated by the elements $\rho(\gamma)-$ id for all $\gamma \in \Gamma$. This is an ideal in the image of the whole group ring. If $V$ is a module over a ring $R$ and the action is $R$-linear, we obviously have $J(V) \subset \operatorname{End}_{R}(V)$. But note that in general $J(V)$ is not an $R$-submodule.

For the adjoint representations of $\tilde{G}$ and $G$ these ideals are roughly characterized in [16] Sect. 4. The following proposition lists a few special consequences. The respective representations and subquotients were defined in Section 1. In (a) and (b) we deal with a reducible representation and look - in terms of a suitable basis - at the matrices of strictly block triangular form. For example, we identify $\operatorname{Hom}_{F}\left(\tilde{M}_{G}, \tilde{K}_{G}\right)$ with a subspace of $\operatorname{End}_{F}\left(\tilde{L}_{G}\right)$ in the obvious way. Assumption 0.1 and [16] Thm. 4.4 imply:

Proposition 4.3. Suppose that $F$ is a field. There exists a finitely generated subring $R \subset F$, such that $F$ is either equal to $\operatorname{Quot}(R)$ or a totally inseparable extension of $\operatorname{Quot}(R)$ of prime degree, and:

(a) $J\left(\tilde{L}_{G}\right) \cap \operatorname{Hom}_{F}\left(\tilde{M}_{G}, \tilde{K}_{G}\right)$ is an $R$-module of finite type which generates $\operatorname{Hom}_{F}\left(\tilde{M}_{G}, \tilde{K}_{G}\right)$ as vector space over $\operatorname{Quot}(R)$.

(b) $J\left(M_{G}\right) \cap \operatorname{Hom}_{F}\left(M_{G} / \tilde{M}_{G}, \tilde{M}_{G}\right)$ is an R-module of finite type which generates $\operatorname{Hom}_{F}\left(M_{G} / \tilde{M}_{G}, \tilde{M}_{G}\right)$ as vector space over $\operatorname{Quot}(R)$.

(c) $J\left(\tilde{M}_{G}\right)$ is an $R$-module of finite type such that $B\left(\tilde{M}_{G}\right):=\operatorname{Quot}(R) \cdot J\left(\tilde{M}_{G}\right)$ is either

(i) equal to $\operatorname{End}_{F}\left(\tilde{M}_{G}\right)$, or

(ii) a central simple algebra over $E:=\left\{x^{2} \mid x \in F\right\}$, where $\operatorname{char}(F)=2$ and the root system of $G$ has type $C_{n}$ for some $n \geq 1$, and

$$
B\left(\tilde{M}_{G}\right) \otimes_{E} F \stackrel{\sim}{\longrightarrow} \operatorname{End}_{F}\left(\tilde{M}_{G}\right)
$$

\section{Weak approximation I}

In this section we apply the results of [16] to simultaneous approximation at a finite set of places. For ease of notation, by a place $v$ of $F$ we mean a place of one of its simple summands. The corresponding local field is denoted $F_{v}$. Observe that we always have a natural homomorphism $F \rightarrow F_{v}$ with dense image, which is injective if and only if $F$ is already a field. The set of archimedean places of $F$ is denoted $S_{\infty}$. For $v \notin S_{\infty}$ we let $\mathscr{O}_{v} \subset F_{v}$ denote the corresponding ring of integers.

For any finite set of places $V$ we set $F_{V}:=\bigoplus_{v \in V} F_{v}$ and abbreviate $G_{V}:=$ $G \times{ }_{F} F_{V}$. Let $\Gamma_{V}$ denote the closure of the image of $\Gamma$ in $G\left(F_{V}\right)=\prod_{v \in V} G\left(F_{v}\right)$. Then the triple $\left(F_{V}, G_{V}, \Gamma_{V}\right)$ satisfies Assumption 3.1 for the local case. 
Proposition 5.1. $\left(F_{V}, G_{V}, \Gamma_{V}\right)$ is minimal.

Proof. For any weak quasi-model $\left(E_{V}, H_{V}, \varphi\right)$ of $\left(F_{V}, G_{V}, \Gamma_{V}\right)$ we must prove $E_{V}=F_{V}$ and that $\varphi$ is an isomorphism. For this we first exploit Proposition 4.2. Let $F^{\prime}, F_{V}^{\prime}$, and $E_{V}^{\prime}$ be obtained from $F, F_{V}$, and $E_{V}$ by doubling all simple summands where the respective algebraic group is non-standard. With $E_{\alpha}$ as in the preceding section, we have a diagram

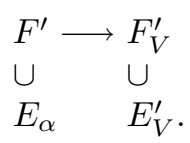

As the representation $\alpha \circ \varphi$ descends to $E_{V}^{\prime}$, all its traces lie in $E_{V}^{\prime}$. Thus the missing homomorphism $E_{\alpha} \rightarrow E_{V}^{\prime}$ also exists, making the diagram commutative. Proposition 4.2 implies that the inclusion $E_{\alpha} \subset F^{\prime}$ induces a bijection between simple summands and is a totally inseparable extension there. We claim that the same is true for the inclusion $E_{V}^{\prime} \subset F_{V}^{\prime}$.

To see this we may decompose all these rings according to the characteristic of their constituents, so we may assume that this common characteristic is $p$. Then every simple summand of $F^{\prime}$ is mapped into $E_{\alpha}$ by some Frobenius map $x \mapsto x^{p^{n}}$. Thus for any sufficiently large $n$, the $p^{n}$-th power of every element of $F^{\prime}$ lies in $E_{\alpha}$. Since the image of $F^{\prime}$ in $F_{V}^{\prime}$ is dense, we deduce that the $p^{n}$-th power of every element of $F_{V}^{\prime}$ lies in $E_{V}^{\prime}$. In particular all primitive idempotents of $F_{V}^{\prime}$ are already in $E_{V}^{\prime}$; hence the inclusion $E_{V}^{\prime} \subset F_{V}^{\prime}$ induces a bijection between simple summands. It is also a totally inseparable extension there, as claimed.

Using the claim and Proposition 3.8, we can now project everything to corresponding simple summands of $F, E_{V}$, and $F_{V}$. We may thus assume that they are fields and can distinguish cases. If $G$ is standard and not of type $A_{1}$ in characteristic 2, we have $E_{\alpha}=F^{\prime}=F$ by Proposition 4.2. This implies $E_{V}=F_{V}$. Here $\varphi$ is automatically an isomorphism, as desired.

If $G$ is non-standard, but not of type $C_{n}$ in characteristic 2 , the simple summand $E_{\ell} \subset E_{\alpha}$ associated to the representation $\alpha_{\ell}$ is equal to $F$ by Proposition 4.2. Again this implies $E_{V}=F_{V}$. Suppose that $\varphi$ is a non-standard isogeny. If $\varphi_{0}$ denotes the basic non-standard isogeny of $G$, the facts explained in Section 1 imply

$$
\alpha_{\ell} \circ \varphi \cong \alpha_{s} \circ \varphi_{0} \circ \varphi \cong \alpha_{s} \circ \operatorname{Frob}_{p} \cong \operatorname{Frob}_{p} \circ \alpha_{s} .
$$

This shows that all traces of the form $\operatorname{Tr} \alpha_{\ell}\left(\Gamma_{V}\right)$ are $p$-th powers; hence $E_{\ell} \subset\left\{x^{p} \mid\right.$ $x \in F_{V}$ \}. But this contradicts the fact that $E_{\ell}=F$ is dense in $F_{V}$. Thus $\varphi$ must be an isomorphism, as desired.

It remains to consider the cases $C_{n}$ with $n \geq 1$ in characteristic 2 . Here we employ Proposition 4.3. As the representation of $\Gamma$ on $M_{G} \otimes_{F} F_{V}$ descends to the subfield $E_{V}$, the intersection $J\left(M_{G}\right) \cap \operatorname{Hom}_{F}\left(M_{G} / \tilde{M}_{G}, \tilde{M}_{G}\right)$ must be contained in a model of $\operatorname{Hom}_{F}\left(M_{G} / \tilde{M}_{G}, \tilde{M}_{G}\right) \otimes_{F} F_{V}$ over $E_{V}$. Thus Proposition 4.3 (b) implies 
$F \subset E_{V}$. Since $F$ is dense in $F_{V}$, this shows $E_{V}=F_{V}$. Suppose that $\varphi$ is a nonstandard isogeny. Recall that the representation of $G$ on $M_{G}$ factors through the basic non-standard isogeny $\varphi_{0}$. As in the calculation 5.2 we deduce that $J\left(M_{G}\right)$ is contained in a model of $\operatorname{End}_{F}\left(M_{G}\right) \otimes_{F} F_{V}$ over the subfield $\left\{x^{2} \mid x \in F_{V}\right\}$. This contradicts Proposition 4.3 (b), as before.

Now let $\pi: \tilde{G} \rightarrow G$ denote the fiberwise universal covering, and $\Gamma^{\prime} \subset \tilde{G}(F)$ the generalized commutator group defined in the introduction. Let $\Gamma_{V}^{\prime}$ denote the closure of the image of $\Gamma^{\prime}$ in $\tilde{G}\left(F_{V}\right)$. Equivalently, this is the closure of the subgroup generated by all generalized commutators of $\Gamma_{V}$.

Let $S_{\Gamma}=\coprod_{i=1}^{m} S_{i, \Gamma}$ denote the set of places $v$ for which the image of $\Gamma$ in $G\left(F_{v}\right)$ does not lie in a compact subgroup. If $V$ is disjoint from $S_{\Gamma}$, then $\Gamma_{V}$ is compact, as required in [16]. Proposition 5.1 and [16] Thm. 7.2 imply weak approximation in this case:

Theorem 5.3. For any finite set of places $V$ which is disjoint from $S_{\Gamma}$, the subgroup $\Gamma_{V}^{\prime} \subset \tilde{G}\left(F_{V}\right)=\prod_{v \in V} \tilde{G}\left(F_{v}\right)$ is open.

\section{Weak approximation II}

Now we study $\Gamma_{V}$ and $\Gamma_{V}^{\prime}$ for an arbitrary finite set of places $V$. For any subset $W \subset V$ we identify

$$
G\left(F_{W}\right) \cong G\left(F_{W}\right) \times \prod_{v \in V \backslash W}\{1\} \subset G\left(F_{V}\right)
$$

and similarly with $\tilde{G}$ in place of $G$. We will show in Theorem 6.12 that there is a partition $V=V_{o} \sqcup V_{d}$ such that $\Gamma_{V}^{\prime}$ is composed of an open subgroup in $G\left(F_{V_{o}}\right)$ and a $\underline{d i s c r e t e ~ s u b g r o u p ~ i n ~} G\left(F_{V_{d}}\right)$. A somewhat weaker property is proved for $\Gamma_{V}$ in Theorem 6.15.

Let $V_{\infty}$ be the set of archimedean places in $V$, and $V_{f}:=V \backslash V_{\infty}$ the set of non-archimedean places. In the following we will analyze a suitable open subgroup $\Delta_{V} \subset \Gamma_{V}$. Its projections to the archimedean and non-archimedean factors are denoted $\Delta_{V_{\infty}} \subset \Gamma_{V_{\infty}}$, resp. $\Delta_{V_{f}} \subset \Gamma_{V_{f}}$. The identity component of any topological group $H$ is denoted $H^{\circ}$.

Proposition 6.2. There exists an open subgroup $\Delta_{V} \subset \Gamma_{V}$ such that

(a) $\Delta_{V}=\Delta_{V_{\infty}} \times \Delta_{V_{f}}$,

(b) $\Delta_{V_{\infty}}$ is a connected real Lie group, and

(c) $\Delta_{V_{f}}$ is compact.

Proof. Choose an open compact subgroup $K_{V_{f}} \subset G\left(F_{V_{f}}\right)$, and let $\Theta_{V}$ denote the intersection of $\Gamma_{V}$ with $G\left(F_{V_{\infty}}\right) \times K_{V_{f}}$. Since $K_{V_{f}}$ is compact, the projection 
map $\mathrm{pr}_{1}$ to the first factor is proper. Therefore $\operatorname{pr}_{1}\left(\Theta_{V}\right)$ is a closed subgroup of $G\left(F_{V_{\infty}}\right)$. By a theorem of Cartan, any closed subgroup of a real Lie group is a Lie subgroup ([11] Ch. II Thm. 2.3). Thus the identity component $\operatorname{pr}_{1}\left(\Theta_{V}\right)^{\circ}$ is open in $\operatorname{pr}_{1}\left(\Theta_{V}\right)$. We define $\Delta_{V} \subset \Theta_{V}$ as the inverse image of this identity component and claim that it has the desired properties. Its construction is summarized in the following commutative diagram:

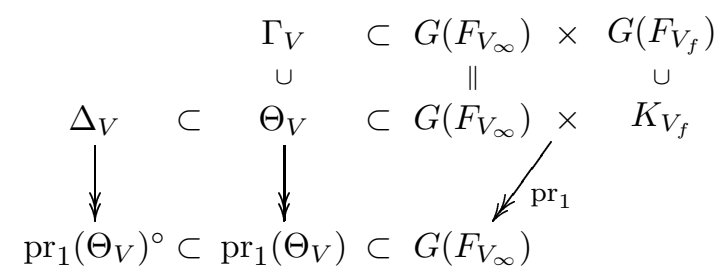

By construction $\Theta_{V}$ and $\Delta_{V}$ are open in $\Gamma_{V}$. Assertion (b) is obvious from the equality $\Delta_{V_{\infty}}=\operatorname{pr}_{1}\left(\Theta_{V}\right)^{\circ}$, and assertion (c) from the inclusion $\Delta_{V_{f}} \subset K_{V_{f}}$. To prove (a) observe that for any open subgroup $K_{V_{f}}^{\prime} \subset K_{V_{f}}$ the intersection $\Delta_{V} \cap\left(G\left(F_{V_{\infty}}\right) \times K_{V_{f}}^{\prime}\right)$ is open in $\Delta_{V}$. Therefore it still maps surjectively to $\operatorname{pr}_{1}\left(\Theta_{V}\right)^{\circ}$. In the limit over $K_{V_{f}}^{\prime}$ we deduce that $\operatorname{pr}_{1}\left(\Theta_{V}\right)^{\circ}$ is in fact contained in $\Delta_{V}$; hence $\Delta_{V}$ decomposes, as desired.

Let $\Delta_{V}$ be as in Proposition 6.2. For later use we observe that its defining properties are preserved on replacing it by any open subgroup.

Proposition 6.3. The subgroup $\Delta_{V}$ is commensurated by $\Gamma_{V}$.

Proof. First consider any open subgroup of $\Delta_{V}$. The properties in Proposition 6.2 imply that it is equal to $\Delta_{V_{\infty}} \times \Delta_{V_{f}}^{\prime}$ for some open subgroup $\Delta_{V_{f}}^{\prime} \subset \Delta_{V_{f}}$. Since $\Delta_{V_{f}}$ is compact, its open subgroup $\Delta_{V_{f}}^{\prime}$ has finite index. This shows that any open subgroup of $\Delta_{V}$ has finite index in $\Delta_{V}$.

Now consider any element $\gamma \in \Gamma_{V}$. As both $\Delta_{V}$ and $\gamma \Delta_{V} \gamma^{-1}$ are open in $\Gamma_{V}$, so is their intersection. Therefore its index in $\Delta_{V}$ is finite. The same argument applies to the index in $\gamma \Delta_{V} \gamma^{-1}$, finishing the proof.

Lemma 6.4. For any place $v \in V$, the image of $\Delta_{V}$ in $G\left(F_{v}\right)$ is either finite or Zariski dense.

Proof. Let $H_{v}$ denote the Zariski closure in $G_{F_{v}}$ of the image of $\Delta_{V}$. Since $\Delta_{V}$ is commensurated by $\Gamma$, so is $H_{v}$. Therefore the identity component $H_{v}^{\circ}$ is normalized by $\Gamma$. As the image of $\Gamma$ in $G\left(F_{v}\right)$ is Zariski dense, the subgroup $H_{v}^{\circ}$ is normal in $G_{F_{v}}$. But this latter group is simple, so $H_{v}$ is either finite or equal to $G_{F_{v}}$.

Let $V_{o}$ be the set of places in $V$ where the image of $\Delta_{V}$ is Zariski dense, and 
set $V_{d}:=V \backslash V_{o}$. Then with the convention of 6.1 we have:

Proposition 6.5. If $\Delta_{V}$ is sufficiently small, it is contained in $G\left(F_{V_{o}}\right)$, and for every $v \in V_{o}$ its image in $G\left(F_{v}\right)$ is Zariski dense.

Next we characterize the archimedean part $\Delta_{V_{\infty}}$. Set $V_{\infty o}:=V_{\infty} \cap V_{o}$ and $V_{f_{o}}:=V_{f} \cap V_{o}$. First we take another look at the adjoint representation of $\Gamma$ :

Lemma 6.6. The $\mathbb{R}[\Gamma]$-modules Lie $G\left(F_{v}\right)$ for all archimedean places of $F$ are non-trivial irreducible and pairwise inequivalent.

Proof. Suppose first that $F$ is a field. There is nothing to prove unless $F$ has characteristic zero, in which case it is a number field. Then Lie $G$ is a non-trivial irreducible representation of $G$ and is therefore equal to $\tilde{M}_{G}$ (cf. Section 1). Thus by Proposition 4.3 (c) the homomorphism $\mathbb{Q}[\Gamma] \longrightarrow \operatorname{End}_{F}(\operatorname{Lie} G)$ is surjective. After tensoring with $\mathbb{R}$ we obtain a surjection

$$
\mathbb{R}[\Gamma] \longrightarrow \operatorname{End}_{F}(\operatorname{Lie} G) \otimes_{\mathbb{Q}} \mathbb{R}=\bigoplus_{v \in S_{\infty}} \operatorname{End}_{F_{v}}\left(\operatorname{Lie} G\left(F_{v}\right)\right),
$$

which clearly implies the desired assertion in the field case.

The map remains surjective in the general case by [16] Thm. 4.4. One can also deduce this from Proposition 4.2, as follows. The field case shows that the image is a subring which surjects to each simple summand. It is therefore a semisimple subalgebra. If it is properly contained in the right hand side, it must lie over the graph of an isomorphism between two simple summands. But then the set of all traces $\operatorname{Tr} \alpha(\Gamma)$ lies over the graph of the corresponding isomorphism $F_{v} \cong F_{w}$ with $v \neq w$, which contradicts Proposition 4.2. Thus the map 6.7 is surjective in general, which proves the lemma.

Proposition 6.8. We have $\Delta_{V_{\infty}}=G\left(F_{V_{\infty}}\right)^{\circ}$.

Proof. Proposition 6.3 implies that the Lie algebra of $\Delta_{V_{\infty}}$ is normalized by $\Gamma$. Thus Lemma 6.6 shows that Lie $\Delta_{V_{\infty}}=\bigoplus_{v \in W}$ Lie $G\left(F_{v}\right)$ for a certain subset $W \subset$ $V_{\infty}$. By integrating we deduce $G\left(F_{W}\right)^{\circ}=\Delta_{V_{\infty}}^{\circ}=\Delta_{V_{\infty}}$. Finally, Proposition 6.5 implies $W=V_{\infty o}$, as desired.

Now we turn to the generalized commutator group.

Proposition 6.9. The group $\Gamma_{V}^{\prime}$ contains an open subgroup of $\tilde{G}\left(F_{V_{o}}\right)$.

Proof. We first deal with the non-archimedean part, setting $V_{f o}:=V_{f} \cap V_{o}$. By Proposition 5.1 the triple $\left(F_{V_{f o}}, G_{V_{f o}}, \Gamma_{V_{f o}}\right)$ is minimal. Proposition 6.5 implies $\Delta_{V_{f}} \subset \Gamma_{V_{f o}}$ and that this subgroup is fiberwise Zariski dense in $G_{V_{f o}}$. Moreover, Proposition 6.3 shows that it is commensurated by $\Gamma_{V_{f o}}$. Since $\Delta_{V_{f}}$ is also 
compact, all the requirements of Proposition 3.10 are fulfilled in this case, and it follows that $\left(F_{V_{f o}}, G_{V_{f o}}, \Delta_{V_{f}}\right)$ is minimal. Now [16] Thm. 7.2 implies that the closure in $\tilde{G}\left(F_{V_{f o}}\right)$ of the generalized commutator group of $\Delta_{V_{f}}$ is open.

Next we consider the closed subgroup $\Gamma_{V}^{\prime} \cap \tilde{G}\left(F_{V_{\infty o}}\right)$. Lemma 6.6 and Proposition 6.8 imply that its Lie algebra is equal to Lie $\tilde{G}\left(F_{V_{\infty} o}\right) \cong \operatorname{Lie} G\left(F_{V_{\infty} o}\right)$. Thus this subgroup is open in $\tilde{G}\left(F_{V_{\infty o}}\right)$. All together we find that the closure in $\tilde{G}\left(F_{V_{o}}\right)$ of the generalized commutator group of $\Delta_{V}$ is open. By construction this open subgroup is contained in $\Gamma_{V}^{\prime}$, as desired.

Next we can split off the non-compact factors from $\Gamma_{V}^{\prime}$. Set $W_{o}:=V_{o} \cap S_{\Gamma}$.

Proposition 6.10. We have $\Gamma_{V}^{\prime}=\tilde{G}\left(F_{W_{o}}\right) \times \Gamma_{V \backslash W_{o}}^{\prime}$.

Proof. For any $v \in W_{o}$ Proposition 6.9 implies that $\Gamma_{V}^{\prime} \cap \tilde{G}\left(F_{v}\right)$ is open in $\tilde{G}\left(F_{v}\right)$. This subgroup is also normalized by $\Gamma$, which by assumption is unbounded at $v$, so by Theorem 2.2 it is equal to $\tilde{G}\left(F_{v}\right)$. It follows that $\tilde{G}\left(F_{W_{o}}\right)=\prod_{v \in W_{o}} \tilde{G}\left(F_{v}\right)$ is contained in $\Gamma_{V}^{\prime}$, and therefore splits off as a direct factor, as desired.

At last, we can turn to the main results of this section.

\section{Lemma 6.11.}

(a) For every $v \in V_{o}$ the subgroup $\Gamma_{V}^{\prime} \cap \tilde{G}\left(F_{v}\right)$ is open in $\tilde{G}\left(F_{v}\right)$ and of finite index in $\Gamma_{v}^{\prime}$.

(b) The subgroup $\Gamma_{V}^{\prime} \cap \tilde{G}\left(F_{V_{o}}\right)$ is open in $\tilde{G}\left(F_{V_{o}}\right)$ and of finite index in $\Gamma_{V_{o}}^{\prime}$.

Proof. The openness in (a) follows from Proposition 6.9. If $v \in W_{o}$, we also have $\Gamma_{V}^{\prime} \cap \tilde{G}\left(F_{v}\right)=\tilde{G}\left(F_{v}\right)=\Gamma_{v}^{\prime}$ by Proposition 6.10 , so the index is 1 . Otherwise $\Gamma_{v}$ is compact; hence so is $\Gamma_{v}^{\prime}$. Since $\Gamma_{V}^{\prime} \cap \tilde{G}\left(F_{v}\right)$ is an open subgroup of $\Gamma_{v}^{\prime}$, its index is finite. This proves (a). Assertion (b) follows from (a) and the inclusions

$$
\prod_{v \in V_{o}}\left(\Gamma_{V}^{\prime} \cap \tilde{G}\left(F_{v}\right)\right) \subset \Gamma_{V}^{\prime} \cap \tilde{G}\left(F_{V_{o}}\right) \subset \Gamma_{V_{o}}^{\prime} \subset \prod_{v \in V_{o}} \Gamma_{v}^{\prime} .
$$

\section{Theorem 6.12.}

(a) $\Gamma_{V}^{\prime} \subset \Gamma_{V_{o}}^{\prime} \times \Gamma_{V_{d}}^{\prime}$ is open of finite index.

(b) $\Gamma_{V_{d}}^{\prime}$ is discrete in $\tilde{G}\left(F_{V_{d}}\right)$.

(c) $\Gamma_{V_{o}}^{\prime}$ is open in $\tilde{G}\left(F_{V_{o}}\right)$.

Proof. Part (c) follows directly from Lemma 6.11 (b). Consider the inclusions

$$
\left(\Gamma_{V}^{\prime} \cap \tilde{G}\left(F_{V_{o}}\right)\right) \times\left(\Gamma_{V}^{\prime} \cap \tilde{G}\left(F_{V_{d}}\right)\right) \subset \Gamma_{V}^{\prime} \subset \Gamma_{V_{o}}^{\prime} \times \Gamma_{V_{d}}^{\prime} .
$$


Here the left hand side is equal to

$$
\Gamma_{V}^{\prime} \cap\left(\left(\Gamma_{V}^{\prime} \cap \tilde{G}\left(F_{V_{o}}\right)\right) \times \tilde{G}\left(F_{V_{d}}\right)\right),
$$

so Lemma 6.11 (b) implies that it is open of finite index in $\Gamma_{V}^{\prime}$. Since $\Gamma_{V}^{\prime}$ surjects to $\Gamma_{V_{d}}^{\prime}$, it follows that $\Gamma_{V}^{\prime} \cap \tilde{G}\left(F_{V_{d}}\right)$ has finite index in $\Gamma_{V_{d}}^{\prime}$.

By Proposition 6.5 we have $\Delta_{V} \cap G\left(F_{V_{d}}\right)=\{1\}$. Since $\Delta_{V}$ is open in $\Gamma_{V}$, it follows that $\Gamma_{V} \cap G\left(F_{V_{d}}\right)$ is a discrete subgroup of $G\left(F_{V_{d}}\right)$. As the universal covering induces a finite-to-one map $\tilde{G}\left(F_{V_{d}}\right) \longrightarrow G\left(F_{V_{d}}\right)$, we deduce that $\Gamma_{V}^{\prime} \cap \tilde{G}\left(F_{V_{d}}\right)$ is a discrete subgroup of $\tilde{G}\left(F_{V_{d}}\right)$. The same now follows for its finite extension $\Gamma_{V_{d}}^{\prime}$. This proves (b). This also implies that both inclusions in 6.13 are open of finite index; whence (a).

\section{Lemma 6.14.}

(a) For every $v \in V_{o}$ the subgroup $\Gamma_{V} \cap G\left(F_{v}\right)$ is open of finite index in $\Gamma_{v}$.

(b) The subgroup $\Gamma_{V} \cap G\left(F_{V_{o}}\right)$ is open of finite index in $\Gamma_{V_{o}}$.

Proof. If $v \in W_{o}$, Proposition 6.10 implies

$$
\pi\left(\tilde{G}\left(F_{v}\right)\right) \subset \Gamma_{V} \cap G\left(F_{v}\right) \subset \Gamma_{v} \subset G\left(F_{v}\right) .
$$

Since $G\left(F_{v}\right) / \pi\left(\tilde{G}\left(F_{v}\right)\right)$ is abelian of finite exponent by Proposition 2.1, and $\Gamma_{v}$ is topologically finitely generated, the index $\left[\Gamma_{v}: \pi\left(\tilde{G}\left(F_{v}\right)\right)\right]$ is finite. Therefore $\Gamma_{V} \cap G\left(F_{v}\right)$ has finite index in $\Gamma_{v}$. As this is a closed subgroup of $G\left(F_{v}\right)$, it is closed of finite index in $\Gamma_{v}$ and therefore open in that group. This proves (a) whenever $v \in W_{o}$. If $v \notin W_{o}$, consider the inclusions

$$
\begin{aligned}
& \pi\left(\Gamma_{V}^{\prime} \cap \tilde{G}\left(F_{v}\right)\right) \subset \Gamma_{V} \cap G\left(F_{v}\right) \subset \quad \Gamma_{v} \quad \subset G\left(F_{v}\right) \\
& \pi\left(\pi^{-1}\left(\Gamma_{v}\right)\right)=\Gamma_{v} \cap \pi\left(\tilde{G}\left(F_{v}\right)\right) .
\end{aligned}
$$

Here $\Gamma_{v} / \Gamma_{v} \cap \pi\left(\tilde{G}\left(F_{v}\right)\right)$ is a subgroup of $G\left(F_{v}\right) / \pi\left(\tilde{G}\left(F_{v}\right)\right)$ which is topologically finitely generated, so as above we see that its order is finite. On the other hand recall that $\Gamma_{v}$ is compact in this case. Since $\pi$ is a finite morphism, the induced map $\tilde{G}\left(F_{v}\right) \longrightarrow G\left(F_{v}\right)$ is proper; hence $\pi^{-1}\left(\Gamma_{v}\right)$ is compact. But $\Gamma_{V}^{\prime} \cap \tilde{G}\left(F_{v}\right)$ is open in $\tilde{G}\left(F_{v}\right)$ by Proposition 6.9. Therefore $\Gamma_{V}^{\prime} \cap \tilde{G}\left(F_{v}\right)$ has finite index in $\pi^{-1}\left(\Gamma_{v}\right)$. Going through the above diagram we find that $\Gamma_{V} \cap G\left(F_{v}\right)$ has finite index in $\Gamma_{v}$. The openness is deduced as in the first case. This proves (a), and (b) follows from this as in the proof of Lemma 6.11.

\section{Theorem 6.15.}

(a) $\Gamma_{V} \subset \Gamma_{V_{o}} \times \Gamma_{V_{d}}$ is open of finite index.

(b) $\Gamma_{V_{d}}$ is discrete in $G\left(F_{V_{d}}\right)$. 
Proof. This follows from Lemma 6.14 in the same way as Theorem 6.12 follows from Lemma 6.11.

\section{Integral model}

From here until the end of Section 12 we will prove many statements at places of $F$ outside some sufficiently large finite set $S$. We will not fix $S$ in advance, but the relevant conditions will be made clear as we go along. This course is justified because all our assertions will be invariant under enlarging $S$.

We assume that $S$ contains all archimedean places and at least one place on every simple summand $F_{i}$. We let $\mathscr{O} \subset F$ denote the ring of elements having no pole outside $S$. Its total ring of quotients is $F$. Thus $\mathscr{S}:=\mathbf{S p e c} \mathscr{O}$ is a finite disjoint union of regular affine curves, arithmetic or geometric, with "function" fields $F_{i}$. Enlarging $S$ is equivalent to replacing $\mathscr{S}$ by an open dense subscheme.

Proposition 7.1. When $S$ is sufficiently large, there exists a group scheme $\mathscr{G} \rightarrow$ $\mathscr{S}$ with generic fibers $G_{i}$, such that $\Gamma \subset \mathscr{G}(\mathscr{O})$.

Here, as elsewhere in the article, we abbreviate $\mathscr{G}(R):=\mathscr{G}(\mathbf{S p e c} R)$ for any $\mathscr{O}$-algebra $R$.

Proof. The problem decomposes with $F$, so we may assume that $F$ is a field. Choose generators $\gamma_{1}, \ldots, \gamma_{r}$ of $\Gamma$ and a faithful representation $\rho: G \hookrightarrow \mathrm{GL}_{n, F}$. Assume that $S$ contains all places where some matrix coefficient of some $\rho\left(\gamma_{i}^{ \pm 1}\right)$ possesses a pole. Let $\mathscr{G}$ be the Zariski closure of $G$ in $\mathrm{GL}_{n} \times \mathscr{S}$. Then $\mathscr{G}(\mathscr{O})$ contains all $\gamma_{i}^{ \pm 1}$, and hence $\Gamma$, as desired.

With $S$ as in Proposition 7.1 we clearly have $S_{\Gamma} \subset S$. Therefore:

Corollary 7.2. The set $S_{\Gamma}$ is finite.

The closed fiber of $\mathscr{G}$ at a place $v \notin S$ will be denoted $G_{v}$.

Proposition 7.3. If $S$ is sufficiently large, all fibers $G_{v}$ are connected adjoint absolutely simple algebraic groups, and the universal covering $\widetilde{\mathscr{G}} \rightarrow \mathscr{G}$ exists over $\mathscr{S}$.

Proof. The problem is local on $\mathscr{S}$, so we may assume that $F$ is a field. Let $\mathscr{G}_{0} \rightarrow$ Spec $\mathbb{Z}$ denote the Chevalley group scheme with split connected adjoint fibers and the same root system as $G$ (see [8] Exp. XXV). Choose a finite extension $F_{0} / F$ such that $G \times{ }_{F} F_{0} \cong \mathscr{G}_{0} \times F_{0}$. Clearly this isomorphism extends to some neighborhood of the generic point. That is, there is a finitely generated ring 
$\mathscr{O} \subset \mathscr{O}_{0} \subset F_{0}$ and, setting $\mathscr{S}_{0}:=\operatorname{Spec} \mathscr{O}_{0}$, an isomorphism

$$
\mathscr{G}_{0} \times \mathscr{S}_{0} \stackrel{\sim}{\longrightarrow} \mathscr{G} \times_{\mathscr{S}} \mathscr{S}_{0}
$$

After shrinking $\mathscr{S}$ the map $\mathscr{S}_{0} \rightarrow \mathscr{S}$ is surjective, which proves the first assertion. The universal covering can be constructed in the same fashion.

Next we extend the representations described in Section 1 to the model $\mathscr{G}$. Let $\mathscr{S}^{\prime} \rightarrow \mathscr{S}$ denote the covering obtained by adjoining a second copy of every connected component where $G_{i}$ is non-standard. In other words $\mathscr{S}^{\prime}=\mathbf{S p e c} \mathscr{O}^{\prime}$ where $\mathscr{O}^{\prime}$ is obtained from $\mathscr{O}$ by doubling the respective direct summands. The total field of quotients of $\mathscr{O}^{\prime}$ is the ring $F^{\prime}$ of 4.1 .

Proposition 7.5. Suppose that $S$ is sufficiently large. Then there exists a representation of $\mathscr{G}$ on a vector bundle on $\mathscr{S}^{\prime}$ which in each fiber is isomorphic to the representation $\alpha$ defined in Section 1. Similarly, there exist representations of $\mathscr{G}$ on vector bundles on $\mathscr{S}$ forming a commutative diagram with exact rows

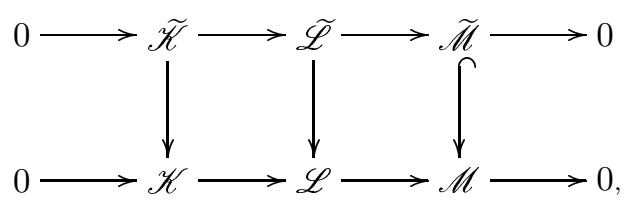

which in every fiber is isomorphic to the diagram 1.1, and where $\widetilde{\mathscr{L}}:=$ Lie $\widetilde{\mathscr{G}}$ and $\mathscr{L}:=\operatorname{Lie} \mathscr{G}$.

Proof. We may assume that $F$ is a field. In the case $\operatorname{char}(F)=0$ the adjoint representation of $G$ is already irreducible and remains so outside a known finite set of primes. Thus after enlarging $S$ accordingly, we simply take $\widetilde{\mathscr{M}}:=\mathscr{M}:=\widetilde{\mathscr{L}} \cong \mathscr{L}$ and $\widetilde{\mathscr{K}}:=\mathscr{K}:=0$. In the case $p:=\operatorname{char}(F)>0$ the whole group scheme $\mathscr{G}$ is already a form of the single group $\mathscr{G}_{0, \mathbb{F}_{p}}$. Since the diagram 1.1 is intrinsically defined, it transfers to any model of $\mathscr{G}_{0, \mathbb{F}_{p}}$ over any scheme, as desired.

Note that with this setup a closed fiber $G_{v}$ is standard if and only if the generic fiber in the connected component of $v$ is standard.

Throughout the rest of the paper we assume that $S$ is so large that $\mathscr{G} \rightarrow \mathscr{S}$ has all the properties in the above propositions.

\section{Genericity}

For every place $v \notin S$ let $\mathfrak{m}_{v} \subset \mathscr{O}$ denote the maximal ideal and $k_{v}:=\mathscr{O} / \mathfrak{m}_{v}$ the residue field at $v$. Let $\bar{\Gamma}_{v}$ denote the image of $\Gamma$ in $\mathscr{G}\left(k_{v}\right)=G_{v}\left(k_{v}\right)$. The aim 
of this section and the next is to identify this subgroup, for almost all $v$. The following result says that $\bar{\Gamma}_{v}$ is sufficiently general in the sense of [15] Sect. 2.

Proposition 8.1. Consider a separated morphism of finite type $\mathscr{T} \rightarrow \mathscr{S}$ and a Zariski closed subgroup scheme $\mathscr{H} \subset \mathscr{G} \times_{\mathscr{S}} \mathscr{T}$ which is fiberwise nowhere dense. Then there exists a finite set of places $S^{\prime}$ containing $S$ such that, for every $v \notin S^{\prime}$ and every geometric point $t$ of $\mathscr{T}$ above $v$, we have $\bar{\Gamma}_{v} \not \subset \mathscr{H}_{t}$.

Proof. Every $\gamma \in \Gamma$ determines a section $\mathscr{T} \longrightarrow \mathscr{G} \times \mathscr{S}$. The inverse image of $\mathscr{H}$ under this section is a Zariski closed subset, consisting of those points $t \in \mathscr{T}$ for which $\mathscr{H}_{t}$ contains the image of $\gamma$. Applying this to a finite set of generators of $\Gamma$ we deduce that the subset of $\mathscr{T}$ where $\mathscr{H}_{t}$ contains the image of $\Gamma$ is Zariski closed.

Its image in $\mathscr{S}$ is therefore constructible ([9] Prop. 9.2.6). We must prove that this image is finite, which reduces to showing that it does not contain any generic point. Suppose it contains the generic point corresponding to the simple summand $F_{i}$. Then there exists a point $t \in \mathscr{T}$ above it where $\Gamma \subset \mathscr{H}_{t}$. But $\mathscr{H}_{t}$ is a proper algebraic subgroup of $G_{i}$ over some extension field. This contradicts the assumption that $\Gamma$ is fiberwise Zariski dense.

For every $v \notin S$ we choose a geometric fiber $G_{\bar{v}}$ of $\mathscr{G}$ above $v$. Recall that $G_{\bar{v}}^{\Phi}$ denotes the group of fixed points of a Frobenius map $\Phi: G_{\bar{v}} \rightarrow G_{\bar{v}}$.

Proposition 8.2. If $S$ is sufficiently large, for every place $v \notin S$ there exists a Frobenius map $\Phi: G_{\bar{v}} \rightarrow G_{\bar{v}}$ such that $\left(G_{\bar{v}}^{\Phi}\right)^{\text {der }}$ is simple and

$$
\left(G_{\bar{v}}^{\Phi}\right)^{\operatorname{der}} \subset \bar{\Gamma}_{v} \subset G_{\bar{v}}^{\Phi} .
$$

Proof. The problem is local on $\mathscr{S}$, so we may assume that $F$ is a field. Let $\mathscr{G}_{0} \rightarrow$ Spec $\mathbb{Z}$ be as in the proof of Proposition 7.3. Consider a separated scheme $\mathscr{T}_{0}$ of finite type over Spec $\mathbb{Z}$ and a fiberwise nowhere dense Zariski closed subgroup scheme $\mathscr{H}_{0} \subset \mathscr{G}_{0} \times \mathscr{T}_{0}$, to be chosen below. Then

$$
\mathscr{H}_{0} \times \mathscr{S}_{0} \subset \mathscr{G}_{0} \times \mathscr{S}_{0} \times \mathscr{T}_{0} \stackrel{7.4}{\cong} \mathscr{G} \times \mathscr{S} \mathscr{S}_{0} \times \mathscr{T}_{0}
$$

is a fiberwise nowhere dense Zariski closed subgroup scheme relative to the base $\mathscr{S}_{0} \times \mathscr{T}_{0}$. Apply Proposition 8.1 to this subgroup scheme and let $S^{\prime}$ denote the resulting finite set of places. For every $v \notin S^{\prime}$ we identify the geometric fiber $G_{\bar{v}}$ with a geometric fiber of $\mathscr{G}_{0}$ via the isomorphism 7.4 . Then by construction we have $\bar{\Gamma}_{v} \not \subset \mathscr{H}_{0, t}$ for every geometric point $t$ of $\mathscr{T}_{0}$ above $\bar{v}$. The existence of the desired Frobenius map now follows from [15] Thm. 0.5, if $\mathscr{H}_{0} \rightarrow \mathscr{T}_{0}$ is suitably chosen. Finally, we replace $S$ by $S^{\prime}$. 


\section{First order approximation}

We will next identify the Frobenius map $\Phi$ in Proposition 8.2, using the trace results of Proposition 4.2. First note that the definition of $\mathscr{O}_{\operatorname{Tr}(\alpha)}$ and Proposition 7.5 imply $\mathscr{O}_{\operatorname{Tr}(\alpha)} \subset \mathscr{O}^{\prime}$.

Proposition 9.1. Suppose that $S$ is sufficiently large.

(a) For any maximal ideal $\mathfrak{m}^{\prime} \subset \mathscr{O}^{\prime}$ we have

$$
\mathscr{O}_{\operatorname{Tr}(\alpha)}+\mathfrak{m}^{\prime}=\mathscr{O}^{\prime}
$$

(b) For any two distinct maximal ideals $\mathfrak{m}_{1}^{\prime}, \mathfrak{m}_{2}^{\prime} \subset \mathscr{O}^{\prime}$ we have

$$
\mathscr{O}_{\operatorname{Tr}(\alpha)}+\mathfrak{m}_{1}^{\prime} \mathfrak{m}_{2}^{\prime}=\mathscr{O}^{\prime}
$$

Proof. Proposition 4.2 implies that the ring extension $\mathscr{O}_{\operatorname{Tr}(\alpha)} \subset \mathscr{O}^{\prime}$ is generically totally inseparable. Geometrically speaking, the dual morphism $\mathscr{S}^{\prime}=$ Spec $\mathscr{O}^{\prime} \longrightarrow$ Spec $\mathscr{O}_{\operatorname{Tr}(\alpha)}$ is generically a totally inseparable covering. After shrinking $\mathscr{S}$ and consequently $\mathscr{S}^{\prime}$, its image is contained in the regular locus. Then the morphism is injective on closed points and induces isomorphisms of residue fields. The assertions follow.

Every place $v \notin S$ where $G_{v}$ is standard determines a unique maximal ideal $\mathfrak{m}_{v}^{\prime} \subset \mathscr{O}^{\prime}$. If $G_{v}$ is non-standard, it determines two distinct maximal ideals $\mathfrak{m}_{v, \ell}^{\prime}$ and $\mathfrak{m}_{v, s}^{\prime} \subset \mathscr{O}^{\prime}$ corresponding to the representations $\alpha_{\ell}$, respectively $\alpha_{s}$.

Proposition 9.2. If $S$ is sufficiently large, for every $v \notin S$ we have

$$
\mathscr{G}\left(k_{v}\right)^{\text {der }} \subset \bar{\Gamma}_{v} \subset \mathscr{G}\left(k_{v}\right) \text {. }
$$

Proof. Let $S$ be at least as large as in Propositions 8.2 and 9.1. We will prove that for $v \notin S$ the Frobenius map $\Phi: G_{\bar{v}} \rightarrow G_{\bar{v}}$ is a standard Frobenius map relative to the finite field $k_{v}$. Let $k_{\bar{v}}$ denote the field of definition of $\bar{v}$.

Suppose first that $G_{v}$, and hence $G_{\bar{v}}$, is standard. Then $\Phi$ is a standard Frobenius map relative to some finite field $k \subset k_{\bar{v}}$. The representation $\alpha$ of $G_{\bar{v}}$ is characterized intrinsically, so it descends to $k$. This implies $\operatorname{Tr} \alpha\left(\bar{\Gamma}_{v}\right) \subset k$ and hence $\mathscr{O}_{\operatorname{Tr}(\alpha)}+\mathfrak{m}_{v}^{\prime} / \mathfrak{m}_{v}^{\prime} \subset k$. Thus, using Proposition 9.1 (a), we find

$$
k_{v}=\frac{\mathscr{O}^{\prime}}{\mathfrak{m}_{v}^{\prime}}=\frac{\mathscr{O}_{\operatorname{Tr}(\alpha)}+\mathfrak{m}_{v}^{\prime}}{\mathfrak{m}_{v}^{\prime}} \subset k
$$

To prove equality note that Proposition 8.2 implies $\left(G_{\bar{v}}^{\Phi}\right)^{\text {der }} \subset \bar{\Gamma}_{v} \subset \mathscr{G}\left(k_{v}\right)$. We can finish by comparing cardinalities. Namely, known size estimates ([4] Prop. 8.6.1, Thm. 14.3.1) imply

$$
\frac{1}{m} \cdot(|k|-1)^{\operatorname{dim} G} \leq\left|\left(G_{\bar{v}}^{\Phi}\right)^{\operatorname{der}}\right| \leq\left|\mathscr{G}\left(k_{v}\right)\right| \leq\left|k_{v}\right|^{\operatorname{dim} G}
$$


where $m$ is the index of the root lattice in the weight lattice of $G_{i}$. If $k \supsetneqq k_{v}$, we have $|k| \geq\left|k_{v}\right|^{2}$, which leads to a contradiction whenever $\left|k_{v}\right|$ is large. Thus after enlarging $S$, if necessary, we must have $k=k_{v}$. This implies $\left(G_{\bar{v}}^{\Phi}\right)^{\text {der }}=\mathscr{G}\left(k_{v}\right)^{\text {der }}$ and hence the desired assertion.

In the non-standard case there are two possibilities. If $\Phi$ is a standard Frobenius, the same proof as above goes through with, say, the representation $\alpha_{\ell}$ in place of $\alpha$. So suppose that $\Phi$ is non-standard. We will show that this cannot happen under our assumptions on $v$. Let $\varphi_{0}: G_{\bar{v}} \rightarrow G_{\bar{v}}$ denote any basic non-standard isogeny with non-zero derivative. The classification of isogenies implies that $\Phi$ is the composite of an odd power $\varphi_{0}^{2 r+1}$ with an automorphism of $G_{\bar{v}}$. Thus

$$
\alpha_{s} \circ \Phi \cong \alpha_{s} \circ \varphi_{0}^{2 r+1} \cong \alpha_{\ell} \circ \varphi_{0}^{2 r} \cong \operatorname{Frob}_{p^{r}} \circ \alpha_{\ell} .
$$

For elements $\bar{\gamma} \in \bar{\Gamma}_{v} \subset G_{\bar{v}}^{\Phi}$ it follows that $\operatorname{Tr} \alpha_{s}(\bar{\gamma})=\operatorname{Tr} \alpha_{\ell}(\bar{\gamma})^{p^{r}}$. Therefore the subring

$$
\frac{\mathscr{O}_{\operatorname{Tr}(\alpha)}+\mathfrak{m}_{v, \ell}^{\prime} \mathfrak{m}_{v, s}^{\prime}}{\mathfrak{m}_{v, \ell}^{\prime} \mathfrak{m}_{v, s}^{\prime}} \subset k_{\bar{v}} \oplus k_{\bar{v}}
$$

lies in the graph of Frob $p_{p^{r}}$. But this contradicts Proposition 9.1 (b).

Proposition 9.3. If $S$ is sufficiently large, for any two distinct places $v, w \notin S$ the image of $\Gamma$ in $\mathscr{G}\left(k_{v}\right) \times \mathscr{G}\left(k_{w}\right)$ contains $\mathscr{G}\left(k_{v}\right)^{\mathrm{der}} \times \mathscr{G}\left(k_{w}\right)^{\mathrm{der}}$.

Proof. Let $S$ be at least as large as before. Consider distinct $v, w \notin S$ and let $\Delta$ denote the image of $\Gamma$ in $\mathscr{G}\left(k_{v}\right) \times \mathscr{G}\left(k_{w}\right)$. We assume that $\Delta^{\text {der }} \neq \mathscr{G}\left(k_{v}\right)^{\text {der }} \times$ $\mathscr{G}\left(k_{w}\right)^{\text {der }}$ and want to derive a contradiction.

Lemma 9.4. If $\left|k_{w}\right|$ is sufficiently large, there exists a field isomorphism $\sigma$ : $k_{w} \stackrel{\sim}{\longrightarrow} k_{v}$ and an isogeny $\varphi: \sigma^{*} G_{v} \longrightarrow G_{w}$, such that $\Delta \subset \operatorname{Graph}(\varphi)$.

Proof. By Proposition 9.2 the subgroup $\Delta^{\text {der }} \varsubsetneqq \mathscr{G}\left(k_{v}\right)^{\text {der }} \times \mathscr{G}\left(k_{w}\right)^{\text {der }}$ surjects to both factors. As these are simple groups, Goursat's lemma shows that $\Delta^{\text {der }}$ is the

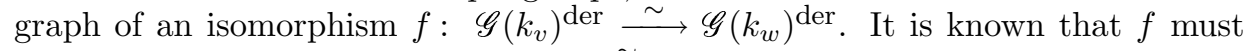
arise from a field isomorphism $\sigma: k_{w} \stackrel{\sim}{\longrightarrow} k_{v}$ and an isogeny $\varphi: \sigma^{*} G_{v} \longrightarrow G_{w}$, if $\left|k_{w}\right|$ is sufficiently large. In fact, by [20] every automorphism of a finite simple group of Lie type is of this form, and the number of exceptional isomorphisms between different finite simple groups of Lie type is finite (see [1]). This shows $\Delta^{\text {der }} \subset \operatorname{Graph}(\varphi)$.

To finish the proof of the lemma, consider any element $\delta=\left(\delta_{v}, \delta_{w}\right) \in \Delta$. Since $\left(\delta_{v}, \varphi\left(\delta_{v}\right)\right) \in \mathscr{G}\left(k_{v}\right) \times \mathscr{G}\left(k_{w}\right)$ normalizes $\Delta^{\text {der }}$, so does the element $\left(1, \delta_{w}^{\prime}\right)$ with $\delta_{w}^{\prime}:=\delta_{w}^{-1} \cdot \varphi\left(\delta_{v}\right)$. Looking at the first component shows that $\left(1, \delta_{w}^{\prime}\right)$ commutes with $\Delta^{\text {der }}$. Projecting to the second component we deduce that $\delta_{w}^{\prime}$ commutes with $\mathscr{G}\left(k_{w}\right)^{\text {der }}$. Since the centralizer of $\mathscr{G}\left(k_{w}\right)^{\text {der }}$ in $\mathscr{G}\left(k_{w}\right)$ is trivial (e.g., by direct 
adaption of [20] 4.4), it follows that $\delta_{w}^{\prime}=1$. We conclude that $\delta_{w}=\varphi\left(\delta_{v}\right)$; whence $\delta \in \operatorname{Graph}(\varphi)$, as desired.

Lemma 9.5. In Lemma 9.4 one can choose $\sigma$ and $\varphi$ such that $d \varphi \neq 0$.

Proof. If $d \varphi=0$, the isogeny $\varphi$ factors through the standard Frobenius

$$
\sigma^{*} G_{v} \longrightarrow \sigma^{*} \operatorname{Frob}_{p}^{*} G_{v} \stackrel{\psi}{\longrightarrow} G_{w} .
$$

We can then replace $\sigma$ by $\operatorname{Frob}_{p} \circ \sigma$ and $\varphi$ by $\psi$, thereby decreasing $\operatorname{deg}(\varphi)$. After iterating this as often as necessary, we have $d \varphi \neq 0$, as desired.

To prove Proposition 9.3, we choose $\sigma$ and $\varphi$ as in the preceding lemmas, noting that the size restriction in 9.4 is satisfied after enlarging $S$. Consider first the standard case. Then $\varphi$ is an isomorphism and we have $\alpha \circ \varphi \cong \sigma^{*} \alpha$. Calculating inside $\mathscr{O}^{\prime} / \mathfrak{m}_{w}^{\prime} \cong k_{w}$, for every $\gamma \in \Gamma$ we have

$$
\sigma^{-1}\left(\operatorname{Tr} \alpha(\gamma) \bmod \mathfrak{m}_{v}^{\prime}\right)=\left(\operatorname{Tr} \alpha(\gamma) \bmod \mathfrak{m}_{w}^{\prime}\right)
$$

This implies

$$
\frac{\mathscr{O}_{\operatorname{Tr}(\alpha)}+\mathfrak{m}_{v}^{\prime} \mathfrak{m}_{w}^{\prime}}{\mathfrak{m}_{v}^{\prime} \mathfrak{m}_{w}^{\prime}} \subset \operatorname{Graph}\left(\sigma^{-1}\right) \subset k_{v} \oplus k_{w} .
$$

But this contradicts Proposition 9.1 (b), as desired.

In the non-standard case, if $\varphi$ is an isomorphism, the same proof applies with $\alpha_{\ell}$ in place of $\alpha$. If $\varphi$ is a non-standard isogeny, we have $\alpha_{s} \circ \varphi \cong \alpha_{\ell}$. This implies, for every $\gamma \in \Gamma$,

$$
\sigma^{-1}\left(\operatorname{Tr} \alpha_{\ell}(\gamma) \bmod \mathfrak{m}_{v, \ell}^{\prime}\right)=\left(\operatorname{Tr} \alpha_{s}(\gamma) \bmod \mathfrak{m}_{w, s}^{\prime}\right) .
$$

Consequently

$$
\frac{\mathscr{O}_{\operatorname{Tr}(\alpha)}+\mathfrak{m}_{v, \ell}^{\prime} \mathfrak{m}_{w, s}^{\prime}}{\mathfrak{m}_{v, \ell}^{\prime} \mathfrak{m}_{w, s}^{\prime}} \subset \operatorname{Graph}\left(\sigma^{-1}\right) \subset k_{v} \oplus k_{w},
$$

again contradicting Proposition 9.1 (b).

\section{Group cohomology}

Next we review a vanishing theorem for $H^{1}$, following Cline, Parshall, and Scott [5], [6]. The representation $\mathscr{M}$ was defined in Proposition 7.5.

Proposition 10.1. If $S$ is sufficiently large, for any $v \notin S$ we have

$$
H^{1}\left(\bar{\Gamma}_{v}, \mathscr{M} \otimes_{\mathscr{O}} k_{v}\right)=0 .
$$


Proof. (Compare [21] Sect. 6.) Consider the composite homomorphism $\widetilde{\mathscr{G}}\left(k_{v}\right) \longrightarrow$ $\mathscr{G}\left(k_{v}\right)^{\text {der }} \subset \bar{\Gamma}_{v}$. Since both the order of its kernel and the index of its image are prime to $\operatorname{char}\left(k_{v}\right)$, it suffices to prove the vanishing theorem for the cohomology of $\widetilde{\mathscr{G}}\left(k_{v}\right)$. We apply the calculations [5] 2.7, 2.8, 3.3 in the split case, resp. [6] 2.2, 2.3, 3.1-2 in the non-split case, to the representation $\mathscr{M} \otimes_{\mathscr{O}} k_{v}=M_{G_{v}}$. Setting $h^{i}:=\operatorname{dim} H^{i}\left(\widetilde{\mathscr{G}}\left(k_{v}\right), M_{G_{v}}\right)$, we obtain the estimate

$$
\begin{aligned}
h^{1}-h^{0} & \leq\left(\text { number of simple root spaces in } M_{G_{v}}\right) \\
& -\left(\text { multiplicity of the weight } 0 \text { in } M_{G_{v}}\right) .
\end{aligned}
$$

provided that $\left|k_{v}\right|>9$. This last condition is satisfied after enlarging $S$.

The terms on the right hand side of 10.2 turn out to be equal. Namely, in the standard case we have $M_{G_{v}}=\operatorname{Lie} G_{v}$, so they are both equal to the rank of $G_{v}$. In the non-standard case the number of simple root spaces in $M_{G_{v}}$ is just the number of simple long roots; hence it is given by the following table:

$$
\begin{array}{l|c|c|c}
B_{n} & C_{n} & F_{4} & G_{2} \\
\hline n-1 & 1 & 2 & 1
\end{array}
$$

The multiplicity of the weight 0 in $M_{G_{v}}$ can be determined with the help of [16] Prop. 1.11 (c). Namely, in the $B_{n}$ case $\alpha_{s}$ is the pullback of the standard representation of dimension $2 \ell$ under the basic isogeny $\varphi_{0}: G_{v} \rightarrow G_{v}^{\sharp} \cong \mathrm{Sp}_{2 \ell}$. Thus it does not contain the weight 0 at all. From [loc. cit.] we deduce that the weight 0 occurs in $\operatorname{Ker}\left(d \varphi_{0}\right)$ with multiplicity 1 ; and hence in $M_{G_{v}}=\operatorname{Coker}\left(d \varphi_{0}\right)$ with multiplicity $n-1$, as desired. Dually, in the $C_{n}$ case $\alpha_{\ell}$ is the composite of the standard representation of $\mathrm{Sp}_{2 \ell}$ with Frob 2 , so it does not possess the weight 0 . Thus the desired multiplicity is equal to $\operatorname{dim}\left(M_{G_{v}} / \tilde{M}_{G_{v}}\right)=1$ in this case. Finally, in the $F_{4}$ and $G_{2}$ case the multiplicity must be half the rank of $G_{v}$ by symmetry, since $\alpha_{\ell} \cong \alpha_{s} \circ \varphi_{0}$.

In all cases the right hand side of 10.2 vanishes, so that $h^{1} \leq h^{0}$. But we also have $h^{0}=0$ (cf. Proposition 12.1 below); hence $h^{1}=0$, as desired.

\section{Second order approximation}

Having clarified the situation modulo $\mathfrak{m}_{v}$, we will now study the image of $\Gamma$ modulo $\mathfrak{m}_{v}^{2}$. Consider the natural short exact sequence

$$
0 \longrightarrow \mathscr{L} \otimes_{\mathscr{O}} \mathfrak{m}_{v} / \mathfrak{m}_{v}^{2} \longrightarrow \mathscr{G}\left(\mathscr{O} / \mathfrak{m}_{v}^{2}\right) \stackrel{\kappa}{\longrightarrow} \mathscr{G}\left(k_{v}\right) \longrightarrow 1
$$

For the moment we consider only the following quotients, where $\mathscr{K}$ is as in 7.5:

$$
0 \longrightarrow \mathscr{M} \otimes_{\mathscr{O}} \mathfrak{m}_{v} / \mathfrak{m}_{v}^{2} \longrightarrow \frac{\mathscr{G}\left(\mathscr{O} / \mathfrak{m}_{v}^{2}\right)}{\mathscr{K} \otimes_{\mathscr{O}} \mathfrak{m}_{v} / \mathfrak{m}_{v}^{2}} \longrightarrow \mathscr{G}\left(k_{v}\right) \longrightarrow 1
$$


Let $\check{\Gamma}_{v}$ denote the image of $\Gamma$ in the middle term of this sequence. We will prove:

Proposition 11.3. If $S$ is sufficiently large, for every $v \notin S$ we have

$$
\check{\Gamma}_{v} \cap\left(\mathscr{M} \otimes_{\mathscr{O}} \mathfrak{m}_{v} / \mathfrak{m}_{v}^{2}\right) \neq 0
$$

To show this, we reduce to the case that $F$ is a field. We set $p:=\operatorname{char}\left(k_{v}\right)$ and distinguish the cases:

(a) $p=0$ in $\mathscr{O} / \mathfrak{m}_{v}^{2}$,

(b) $p \neq 0$ in $\mathscr{O} / \mathfrak{m}_{v}^{2}$.

In the first case we can choose an isomorphism $\mathscr{O} / \mathfrak{m}_{v}^{2} \cong k_{v}[\varepsilon]$ with $\varepsilon^{2}=0$. The information of Section 4 yields:

Proposition 11.4. If $S$ is sufficiently large, for every $v \notin S$ in case (a) the representation of $\Gamma$ on the $k_{v}[\varepsilon]$-module $\mathscr{M} \otimes_{\mathscr{O}} k_{v}[\varepsilon]$ is not the extension of scalars of a representation over $k_{v}$.

Proof. Assume first that we are in the case (i) of Proposition 4.3 (c). Note that the definition of $J\left(\tilde{M}_{G}\right)$ together with Proposition 7.5 implies

$$
J\left(\tilde{M}_{G}\right) \subset \operatorname{End}_{\mathscr{O}}(\widetilde{\mathscr{M}}) .
$$

With $R$ as in (4.3) this inclusion becomes an isomorphism over $\operatorname{Quot}(R)$. The dual morphism $\mathscr{S}=\operatorname{Spec} \mathscr{O} \longrightarrow \operatorname{Spec} R$ is generically finite, and both sides in 11.5 are modules of finite type. As this inclusion is an isomorphism generically on $\operatorname{Spec} R$, it is an isomorphism over an open dense subset of Spec $R$. It follows that the natural map

$$
J\left(\tilde{M}_{G}\right) \longrightarrow \operatorname{End}_{\mathscr{O}}(\widetilde{\mathscr{M}}) \otimes_{\mathscr{O}} k_{v}[\varepsilon]
$$

is surjective for almost all $v$. Thus the representation of $\Gamma$ on $\widetilde{\mathscr{M}} \otimes_{\mathscr{O}} k_{v}[\varepsilon]$ cannot descend to $k_{v}$. As this is a subrepresentation of $\mathscr{M} \otimes_{\mathscr{O}} k_{v}[\varepsilon]$, the proposition follows in this case.

In the case (ii) of Proposition 4.3 (c) we have $\operatorname{char}(F)=2$, and $G$ has type $C_{n}$ for some $n \geq 1$. Therefore $\widetilde{\mathscr{M}} \varsubsetneqq \mathscr{M}$ (cf. Section 1). In the notation of Proposition $4.3(\mathrm{~b})$ the inclusion

$$
\begin{aligned}
J\left(M_{G}\right) \cap \operatorname{Hom}_{F}\left(M_{G} / \tilde{M}_{G}, \tilde{M}_{G}\right) & =J\left(M_{G}\right) \cap \operatorname{Hom}_{\mathscr{O}}(\mathscr{M} / \widetilde{\mathscr{M}}, \widetilde{\mathscr{M}}) \\
& \subset \operatorname{Hom}_{\mathscr{O}}(\mathscr{M} / \widetilde{\mathscr{M}}, \widetilde{\mathscr{M}})
\end{aligned}
$$

becomes an isomorphism over $\operatorname{Quot}(R)$. It follows that the natural map

$$
J\left(M_{G}\right) \cap \operatorname{Hom}_{\mathscr{O}}(\mathscr{M} / \widetilde{\mathscr{M}}, \widetilde{\mathscr{M}}) \longrightarrow \operatorname{Hom}_{\mathscr{O}}(\mathscr{M} / \widetilde{\mathscr{M}}, \widetilde{\mathscr{M}}) \otimes_{\mathscr{O}} k_{v}[\varepsilon]
$$


is surjective for almost all $v$. Again this implies that the representation cannot descend to $k_{v}$.

Proof of Proposition 11.3 in the case (a): Since the algebraic group $G_{v}$ cannot be deformed ([8] Exp. XXIV Prop. 1.21), there exists an isomorphism

$$
\mathscr{G} \times_{\mathscr{S}} \operatorname{Spec} \mathscr{O} / \mathfrak{m}_{v}^{2} \cong G_{v} \times{ }_{\operatorname{Spec} k_{v}} \operatorname{Spec} k_{v}[\varepsilon]
$$

This isomorphism determines a section of $\mathscr{G}\left(k_{v}\right)$ into $\mathscr{G}\left(k_{v}[\varepsilon]\right)=\mathscr{G}\left(\mathscr{O} / \mathfrak{m}_{v}^{2}\right)$, and hence into the middle term of 11.2. On the other hand, if 11.3 fails, the subgroup $\check{\Gamma}_{v}$ is the image of another section over the subgroup $\bar{\Gamma}_{v} \subset \mathscr{G}\left(k_{v}\right)$. Proposition 10.1 implies that all such sections are conjugate. Thus after modifying the isomorphism 11.6 we may assume that these two sections over $\bar{\Gamma}_{v}$ coincide.

If $G_{v}$ is standard, recall that $\mathscr{K}=0$. Otherwise the representation on $\mathscr{M}$ factors through the basic non-standard isogeny $\varphi_{0}: \mathscr{G} \rightarrow \mathscr{G} \sharp$, and we have $\mathscr{K}=$ $\operatorname{Ker}\left(d \varphi_{0}\right)$. In both cases we deduce that the action of $\mathscr{G}\left(\mathscr{O} / \mathfrak{m}_{v}^{2}\right)$ on $\mathscr{M} \otimes_{\mathscr{O}} \mathscr{O} / \mathfrak{m}_{v}^{2}$ factors through the group in the middle of 11.2.

We can now conclude that the action of $\check{\Gamma}_{v}$ on $\mathscr{M} \otimes_{\mathscr{O}} k_{v}[\varepsilon]$ has the same image as the action of $\mathscr{G}\left(k_{v}\right)$. Therefore this action descends to $k_{v}$, contradicting Proposition 11.4.

Proof of Proposition 11.3 in the case (b): This is the unequal characteristic case, so we have $\mathscr{K}=0$, and $\check{\Gamma}_{v}$ is the image of $\Gamma$ in $\mathscr{G}\left(\mathscr{O} / \mathfrak{m}_{v}^{2}\right)$. Recall also that $\mathscr{G}\left(k_{v}\right)^{\text {der }} \subset \bar{\Gamma}_{v} \subset \mathscr{G}\left(k_{v}\right)$ by Proposition 9.2.

As $k_{v}$ is a finite field, the closed fiber $G_{v}$ is quasi-split ([2] Ch. V Prop. 16.6). Choose a maximal torus contained in a Borel subgroup, and lift them to a maximal torus and a Borel subgroup $\mathscr{T} \subset \mathscr{B}$ defined over $\mathscr{O} / \mathfrak{m}_{v}^{2}$ (e.g., using [8] Exp. XXVI Th. 3.16 (a)). Consider the pullback $\kappa^{-1}\left(\mathscr{T}\left(k_{v}\right)\right) \subset \mathscr{G}\left(\mathscr{O} / \mathfrak{m}_{v}^{2}\right)$ in 11.1. Its intersection with $\check{\Gamma}_{v}$ is an extension of the group $\mathscr{T}\left(k_{v}\right) \cap \bar{\Gamma}_{v}$ of prime-to- $p$ order with a $p$-group. It therefore possesses a section; i.e., there exists a subgroup $\Theta \subset \check{\Gamma}_{v}$ which maps isomorphically to $\mathscr{T}\left(k_{v}\right) \cap \bar{\Gamma}_{v}$. Applying the same argument to $\mathscr{T}\left(\mathscr{O} / \mathfrak{m}_{v}^{2}\right)$ yields a similar section within $\mathscr{T}\left(\mathscr{O} / \mathfrak{m}_{v}^{2}\right)$. Being two sections from a prime-to- $p$ group within the common $p$-extension $\kappa^{-1}\left(\mathscr{T}\left(k_{v}\right)\right)$, they are conjugate under $\mathscr{L} \otimes_{\mathscr{O}} \mathfrak{m}_{v} / \mathfrak{m}_{v}^{2}$. Thus after replacing $\mathscr{T} \subset \mathscr{B}$ by suitable conjugates, we may assume $\Theta \subset \mathscr{T}\left(\mathscr{O} / \mathfrak{m}_{v}^{2}\right)$.

Let $\mathscr{U} \subset \mathscr{B}$ be the root group associated to the highest root, and consider the subgroup $\kappa^{-1}\left(\mathscr{U}\left(k_{v}\right)\right) \cap \check{\Gamma}_{v}$. On the one hand it surjects to $\mathscr{U}\left(k_{v}\right)$. On the other hand it is normalized by $\Theta$. If $\left|k_{v}\right|$ is sufficiently large, all roots are distinguished from each other and from the weight 0 by the action of $\mathscr{T}\left(k_{v}\right) \cap \mathscr{G}\left(k_{v}\right)^{\text {der }}$, and hence by $\Theta$. It follows that $\mathscr{U}\left(\mathscr{O} / \mathfrak{m}_{v}^{2}\right) \cap \check{\Gamma}_{v}$ surjects to $\mathscr{U}\left(k_{v}\right)$. In view of assumption (b) this implies Lie $\mathscr{U} \otimes_{\mathscr{O}} \mathfrak{m}_{v} / \mathfrak{m}_{v}^{2} \subset \check{\Gamma}_{v}$. Since Lie $\mathscr{U} \subset \mathscr{L}=\mathscr{M}$ in this case, the proposition follows. 


\section{Higher order approximation}

Recall that $\mathscr{O}_{v}$ denotes the completed local ring at $v$. We will now give a criterion for a subgroup of $\widetilde{\mathscr{G}}\left(\mathscr{O}_{v}\right)$ to be dense, based on its behavior modulo $\mathfrak{m}_{v}^{2}$. First we show that the action of $\Gamma$ on the Lie algebra of a closed fiber is big in the following sense.

Proposition 12.1. If $S$ is sufficiently large, for every $v \notin S$ we have:

(a) There is no $\Gamma$-invariant non-zero proper additive subgroup of $\widetilde{\mathscr{M}} \otimes_{\mathscr{O}} k_{v}$.

(b) There is no $\Gamma$-invariant proper additive subgroup of $\widetilde{\mathscr{L}} \otimes_{\mathscr{O}} k_{v}$ whose image in $\widetilde{\mathscr{M}} \otimes_{\mathscr{O}} k_{v}$ is non-zero.

(c) Any non-zero $\Gamma$-invariant additive subgroup of $\mathscr{M} \otimes_{\mathscr{O}} k_{v}$ contains $\widetilde{\mathscr{M}} \otimes_{\mathscr{O}} k_{v}$.

Proof. For (a) it suffices to show that the natural map

$$
J\left(\tilde{M}_{G}\right) \longrightarrow \operatorname{End}_{\mathscr{O}}(\widetilde{\mathscr{M}}) \otimes_{\mathscr{O}} k_{v} \cong \operatorname{End}_{k_{v}}\left(\widetilde{\mathscr{M}} \otimes_{\mathscr{O}} k_{v}\right)
$$

is surjective for almost all $v$. In the case (i) of Proposition 4.3 (c) this follows already from the proof of Proposition 11.4. Suppose we have the case (ii) of 4.3 (c). Then $J\left(\tilde{M}_{G}\right)$ is an order in a central simple algebra over $E:=\left\{x^{2} \mid x \in F\right\}$ and becomes isomorphic to $\operatorname{End}_{F}\left(\tilde{M}_{G}\right)$ after tensoring with $F$. We also have $\operatorname{Quot}(R)=E$, so putting $\sqrt{R}:=\left\{x \in F \mid x^{2} \in R\right\}$ we obtain a natural map

$$
J\left(\tilde{M}_{G}\right) \otimes_{R} \sqrt{R} \longrightarrow \operatorname{End}_{\mathscr{O}}(\widetilde{\mathscr{M}}) .
$$

By construction this induces an isomorphism over an open dense subset of Spec $R$, and hence over the residue fields at almost all closed points. Since tensoring with $\sqrt{R}$ over $R$ has no effect over the residue fields, the map 12.2 is still surjective for almost all $v$. This implies (a).

For (b) we first note $J\left(\tilde{L}_{G}\right) \subset \operatorname{End}_{\mathscr{O}}(\widetilde{\mathscr{L}})$. With $R$ as in 4.3 (a) the inclusion

$$
\begin{aligned}
J\left(\tilde{L}_{G}\right) \cap \operatorname{Hom}_{F}\left(\tilde{M}_{G}, \tilde{K}_{G}\right) & =J\left(\tilde{L}_{G}\right) \cap \operatorname{Hom}_{\mathscr{O}}(\widetilde{\mathscr{M}}, \widetilde{\mathscr{K}}) \\
& \subset \operatorname{Hom}_{\mathscr{O}}(\widetilde{\mathscr{M}}, \widetilde{\mathscr{K}})
\end{aligned}
$$

becomes an isomorphism over $\operatorname{Quot}(R)$. It follows that the natural map

$$
J\left(\tilde{L}_{G}\right) \cap \operatorname{Hom}_{\mathscr{O}}(\widetilde{\mathscr{M}}, \widetilde{\mathscr{K}}) \longrightarrow \operatorname{Hom}_{\mathscr{O}}(\widetilde{\mathscr{M}}, \widetilde{\mathscr{K}}) \otimes_{\mathscr{O}} k_{v}
$$

is surjective for almost all $v$ (compare the proof of 11.4). Thus the subgroup

$$
\operatorname{Hom}_{k_{v}}\left(\widetilde{\mathscr{M}} \otimes_{\mathscr{O}} k_{v}, \widetilde{\mathscr{K}} \otimes_{\mathscr{O}} k_{v}\right) \subset \operatorname{End}_{k_{v}}\left(\widetilde{\mathscr{L}} \otimes_{\mathscr{O}} k_{v}\right)
$$

is in the image of the group ring $\mathbb{Z}[\Gamma]$. Any $\Gamma$-invariant additive subgroup of $\widetilde{\mathscr{L}} \otimes_{\mathscr{O}} k_{v}$ with non-trivial image in $\widetilde{\mathscr{M}} \otimes_{\mathscr{O}} k_{v}$ must therefore contain $\widetilde{\mathscr{K}} \otimes_{\mathscr{O}} k_{v}$. 
By (a) it also surjects to $\widetilde{\mathscr{M}} \otimes_{\mathscr{O}} k_{v}$. It is therefore equal to $\widetilde{\mathscr{L}} \otimes_{\mathscr{O}} k_{v}$, proving (b). In the same way one proves (c), using 4.3 (b) in place of 4.3 (a).

Proposition 12.3. Suppose that $S$ is sufficiently large. Consider $v \notin S$ and a subgroup $\Delta \subset \widetilde{\mathscr{G}}\left(\mathscr{O}_{v}\right)$ which is normalized by $\Gamma$ and whose image in $\mathscr{G}\left(k_{v}\right)$ is $\mathscr{G}\left(k_{v}\right)^{\text {der }}$. Then $\Delta$ is dense in $\widetilde{\mathscr{G}}\left(\mathscr{O}_{v}\right)$.

The proof will occupy the rest of this section. By Proposition 3.8 we may assume that $F$ is a field. We must first set up the framework for successive approximations. The subgroups

$$
H_{i}:=\operatorname{Ker}\left(\mathscr{G}\left(\mathscr{O}_{v}\right) \rightarrow \mathscr{G}\left(\mathscr{O} / \mathfrak{m}_{v}^{i}\right)\right)
$$

for all integers $i \geq 0$ form a cofinal descending sequence of open compact normal subgroups of $H_{0}=\mathscr{G}\left(\mathscr{O}_{v}\right)$. The graded pieces of degrees $i \geq 1$ are related to the Lie algebra by canonical isomorphisms

$$
H_{i} / H_{i+1} \cong \mathscr{L} \otimes_{\mathscr{O}} \mathfrak{m}_{v}^{i} / \mathfrak{m}_{v}^{i+1}
$$

Similarly, for every $i \geq 0$ we let

$$
\tilde{H}_{i}:=\operatorname{Ker}\left(\widetilde{\mathscr{G}}\left(\mathscr{O}_{v}\right) \rightarrow \widetilde{\mathscr{G}}\left(\mathscr{O} / \mathfrak{m}_{v}^{i}\right)\right)
$$

and for every $i \geq 1$ we have

$$
\tilde{H}_{i} / \tilde{H}_{i+1} \cong \widetilde{\mathscr{L}} \otimes_{\mathscr{O}} \mathfrak{m}_{v}^{i} / \mathfrak{m}_{v}^{i+1}
$$

The isogeny $\pi: \widetilde{\mathscr{G}} \rightarrow \mathscr{G}$ induces a natural commutative diagram

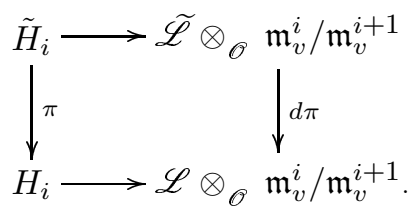

The generalized commutator maps $\widetilde{\mathscr{G}} \times \widetilde{\mathscr{G}} \rightarrow \mathscr{G} \times \widetilde{\mathscr{G}} \rightarrow \mathscr{G} \times \mathscr{G} \rightarrow \widetilde{\mathscr{G}}$ induce for any $i, j \geq 1$ a commutative diagram 


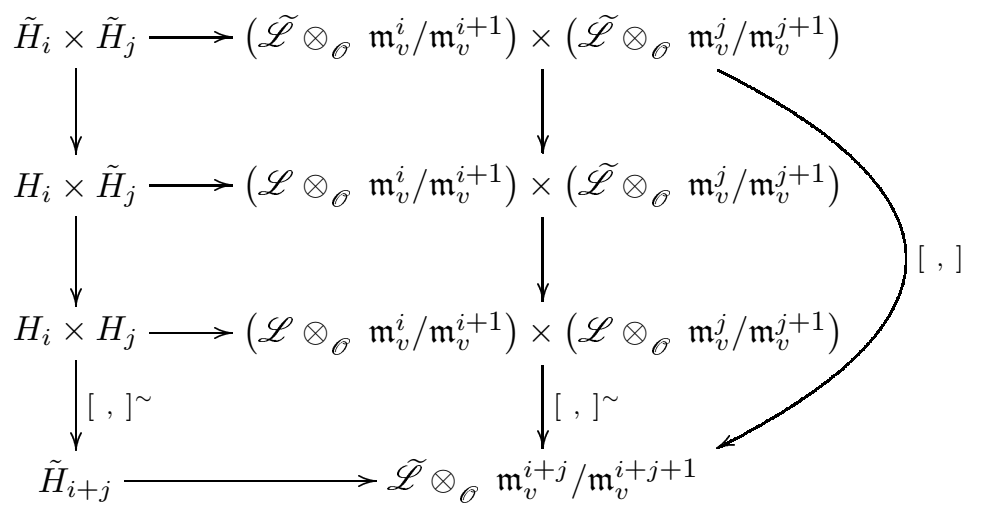

involving the generalized Lie bracket described in Section 1.

Suppose now that $S$ is as large as required by all propositions so far. We claim that Proposition 12.3 holds with this choice. Take $\Delta \subset \widetilde{\mathscr{G}}\left(\mathscr{O}_{v}\right)$ as in 12.3 . It suffices to prove that the map

$$
\Delta \longrightarrow \tilde{\mathscr{G}}\left(\mathscr{O} / \mathfrak{m}_{v}^{i}\right) \cong \tilde{H}_{0} / \tilde{H}_{i}
$$

is surjective for every $i \geq 1$. By induction this reduces to the following assertion.

Proposition 12.4. Under the above assumptions, for every $i \geq 0$ the map $\Delta \cap$ $\tilde{H}_{i} \longrightarrow \tilde{H}_{i} / \tilde{H}_{i+1}$ is surjective.

This will be proved in a sequence of lemmas.

Lemma 12.5. Proposition 12.4 is true for $i=0$.

Proof. It is known that $\tilde{H}_{0} / \tilde{H}_{1} \cong \widetilde{\mathscr{G}}\left(k_{v}\right) \rightarrow \mathscr{G}\left(k_{v}\right)^{\text {der }}$ is a perfect central extension ([7] Thm. 5.27). Since $\Delta \rightarrow \mathscr{G}\left(k_{v}\right)^{\text {der }}$ is surjective by assumption, the lemma follows.

Lemma 12.6. Proposition 12.4 is true for $i=1$.

Proof. Rephrasing Proposition 11.3, the image of $\Gamma \cap H_{1}$ in $\mathscr{M} \otimes_{\mathscr{O}} \mathfrak{m}_{v} / \mathfrak{m}_{v}^{2}$ is nonzero. Thus by Proposition 12.1 (c) this image contains $\widetilde{\mathscr{M}} \otimes_{\mathscr{O}} \mathfrak{m}_{v} / \mathfrak{m}_{v}^{2}$. To transfer this information to $\Delta$, recall that $\Gamma$ normalizes $\Delta$ by assumption. Therefore all generalized commutators of the form $\left[\Gamma \cap H_{1}, \Delta\right]^{\sim}$ are contained in $\Delta \cap \tilde{H}_{1}$. It follows that the image of $\Delta \cap \tilde{H}_{1}$ in $\widetilde{\mathscr{M}} \otimes_{\mathscr{O}} \mathfrak{m}_{v} / \mathfrak{m}_{v}^{2}$ contains all commutators of $\Delta$ with $\widetilde{\mathscr{M}} \otimes_{\mathscr{O}} \mathfrak{m}_{v} / \mathfrak{m}_{v}^{2}$. By assumption this action of $\Delta$ coincides with that 
of $\mathscr{G}\left(k_{v}\right)^{\text {der }}$ and is therefore non-trivial. This shows that the image of $\Delta \cap \tilde{H}_{1}$ in $\widetilde{\mathscr{M}} \otimes_{\mathscr{O}} \mathfrak{m}_{v} / \mathfrak{m}_{v}^{2}$ is non-zero. Now Proposition 12.1 (b) implies that the map $\Delta \cap \tilde{H}_{1} \longrightarrow \tilde{H}_{1} / \tilde{H}_{2} \cong \widetilde{\mathscr{L}} \otimes_{\mathscr{O}} \mathfrak{m}_{v} / \mathfrak{m}_{v}^{2}$ is surjective, as desired.

Lemma 12.7. Suppose that $G$ is not of type $C_{n}$ for $n \geq 1$ with $\operatorname{char}(F)=2$. Then Proposition 12.4 is true for all $i \geq 0$.

Proof. By the preceding lemmas and induction on $i$, we may take $i \geq 1$, assume the assertion for all $i^{\prime} \leq i$, and prove it for $i+1$. The inductive assumption implies that the vertical arrow on the left hand side of the following commutative diagram is surjective:

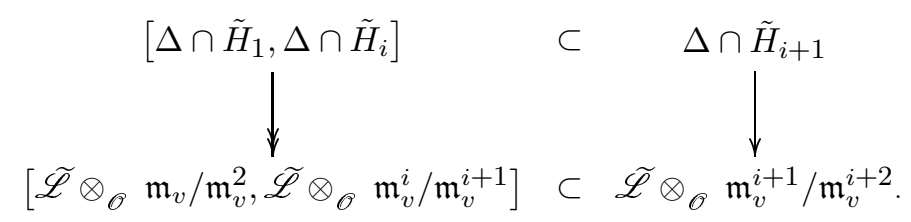

Applying Proposition 1.2 (a) to $\tilde{L}_{G_{v}}=\widetilde{\mathscr{L}} \otimes_{\mathscr{O}} k_{v}$, under the stated assumptions we deduce that the lower inclusion is an equality. It follows that the vertical arrow on the right hand side is surjective, as desired.

In the rest of this section we assume that $G$ has type $C_{n}$ for $n \geq 1$, and that $\operatorname{char}(F)=2$. In this case there is an exact sequence

$$
0 \longrightarrow \widetilde{\mathscr{Z}} \longrightarrow \widetilde{\mathscr{L}} \stackrel{d \pi}{\longrightarrow} \mathscr{L} \longrightarrow \mathscr{M} / \widetilde{\mathscr{M}} \longrightarrow 0
$$

where both $\widetilde{\mathscr{Z}}$ and $\mathscr{M} / \widetilde{\mathscr{M}}$ have rank one (cf. Section 1$)$. This sequence will play a role for certain open compact subgroups. Note first that the map

$$
\tilde{\mathscr{G}}\left(k_{v}\right) \cong \tilde{H}_{0} / \tilde{H}_{1} \longrightarrow H_{0} / H_{1} \cong \mathscr{G}\left(k_{v}\right)
$$

is an isomorphism, since the isogeny $\pi: \widetilde{\mathscr{G}} \rightarrow \mathscr{G}$ is totally inseparable. Therefore we have $\pi^{-1}\left(H_{1}\right)=\tilde{H}_{1}$ inside $\tilde{H}_{0}$. From this we obtain the following commutative diagram with exact rows:

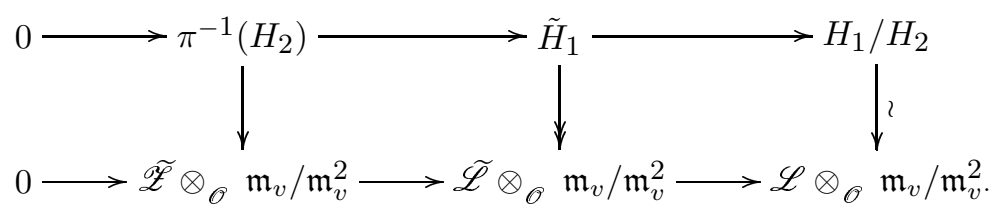

By diagram chasing we find that the leftmost vertical map is surjective. Its kernel is $\tilde{H}_{2}$. Consider the maps indicated by solid arrows in the diagram 


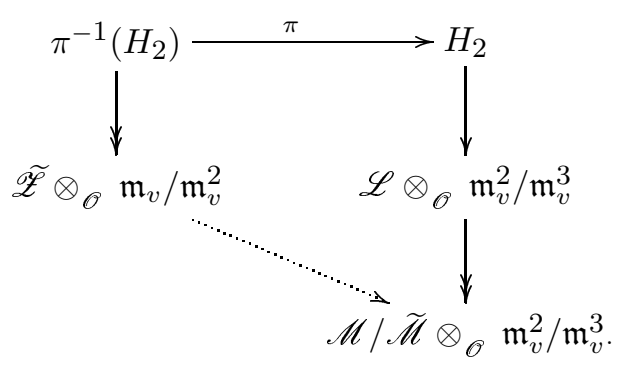

The composite morphism from the upper left corner to the lower right corner restricts to zero on $\tilde{H}_{2}$. Thus it factors through a unique dotted arrow making the diagram commutative.

Lemma 12.10. The dotted arrow in the diagram 12.9 is an isomorphism.

Proof. Since $\widetilde{\mathscr{G}}$ has type $C_{n}$, its root lattice has index 2 in its weight lattice. On dualizing we can thus find a primitive cocharacter $\lambda: \mathbb{G}_{m} \rightarrow \mathscr{G}$, whose square lifts to a cocharacter $\tilde{\lambda}: \mathbb{G}_{m} \rightarrow \widetilde{\mathscr{G}}$, but which does not itself lift. In other words we have a commutative diagram

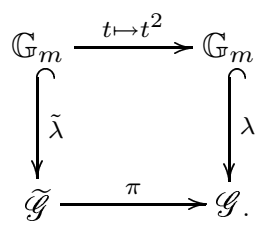

Taking Lie algebras, we obtain a commutative diagram with exact rows

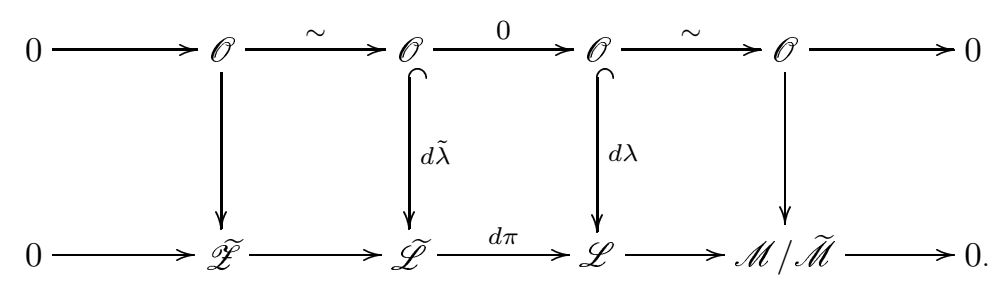

Here the leftmost vertical map is an isomorphism for dimension reasons. The fact that $\lambda$ is not congruent modulo 2 to a cocharacter coming from $\widetilde{\mathscr{G}}$ implies $\operatorname{im}(d \lambda) \not \subset \operatorname{im}(d \pi)$. Thus again for dimension reasons the rightmost vertical map is an isomorphism. Taking $\mathscr{O}_{v}$-valued points in the respective groups we find a commutative diagram 


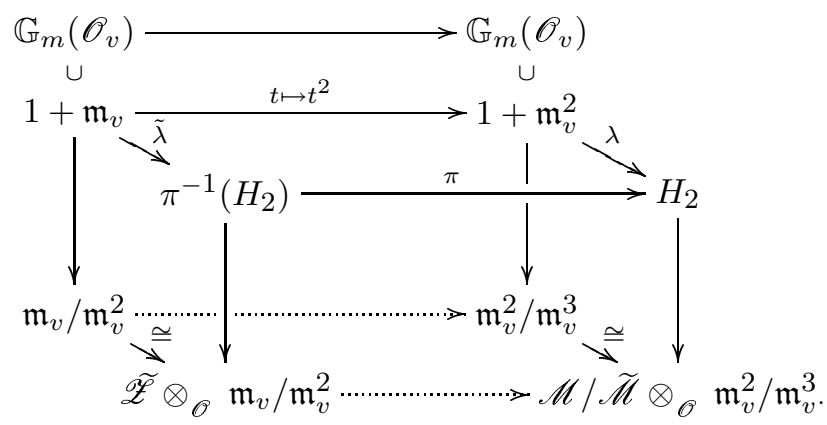

Here the vertical maps in the back are defined by $1+x \mapsto x$. Thus the dotted arrow in the back is given by $x \mapsto(1+x)^{2}-1=x^{2}+2 x$. Since 2 vanishes in $\mathscr{O}$, this is just the Frobenius map $x \mapsto x^{2}$, which clearly induces an isomorphism. Therefore the dotted arrow in front is an isomorphism, as desired.

Lemma 12.11. The composite map $\pi(\Delta) \cap H_{2} \longrightarrow \mathscr{M} / \mathscr{\mathscr { M }} \otimes_{\mathscr{O}} \mathfrak{m}_{v}^{2} / \mathfrak{m}_{v}^{3}$ is non-zero.

Proof. Consider the commutative diagram

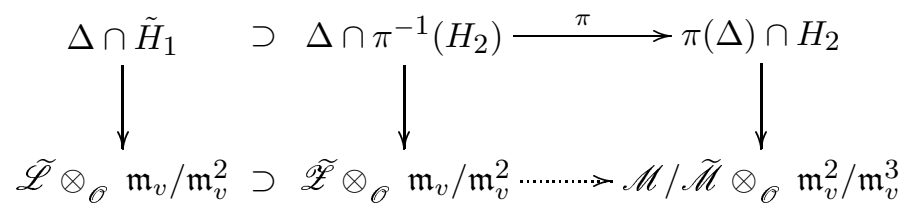

deduced from the diagrams 12.8 and 12.9. The leftmost vertical map is surjective by Lemma 12.6. From 12.8 one deduces that the left half is cartesian; hence the middle map is also surjective. The dotted arrow is bijective by Lemma 12.10. Thus the rightmost vertical map is surjective.

Lemma 12.12. Proposition 12.4 is true for $i=2$.

Proof. We proceed as in the proof of Lemma 12.6. Lemma 12.11 implies that the image of $\pi(\Delta) \cap H_{2}$ in $\mathscr{M} \otimes_{\mathscr{O}} \mathfrak{m}_{v}^{2} / \mathfrak{m}_{v}^{3}$ is non-zero. By Proposition 12.1 (c) this image therefore contains $\widetilde{\mathscr{M}} \otimes_{\mathscr{O}} \mathfrak{m}_{v}^{2} / \mathfrak{m}_{v}^{3}$. All generalized commutators of the form $\left[\pi(\Delta) \cap H_{2}, \Gamma\right] \sim$ are contained in $\Delta \cap \tilde{H}_{2}$, and their images in $\widetilde{\mathscr{M}} \otimes_{\mathscr{O}} \mathfrak{m}_{v}^{2} / \mathfrak{m}_{v}^{3}$ comprise all commutators of $\Gamma$ with $\widetilde{\mathscr{M}} \otimes_{\mathscr{O}} \mathfrak{m}_{v}^{2} / \mathfrak{m}_{v}^{3}$. As $\mathscr{G}\left(k_{v}\right)^{\text {der }}$ and hence $\Gamma$ acts non-trivially on this group, the map $\Delta \cap \tilde{H}_{2} \longrightarrow \widetilde{\mathscr{M}} \otimes_{\mathscr{O}} \mathfrak{m}_{v}^{2} / \mathfrak{m}_{v}^{3}$ must be nonzero. Now Proposition 12.1 (b) implies that the map $\Delta \cap \tilde{H}_{2} \longrightarrow \widetilde{\mathscr{L}} \otimes_{\mathscr{O}} \mathfrak{m}_{v}^{2} / \mathfrak{m}_{v}^{3}$ is surjective, as desired. 
Lemma 12.13. Proposition 12.4 is true for all $i \geq 0$.

Proof. (Compare Lemma 12.7.) By the preceding lemmas and induction on $i$, we may take $i \geq 2$, assume the assertion for all $i^{\prime} \leq i$, and prove it for $i+1$. By Lemma 12.11 we may choose an element $\delta \in \pi(\Delta) \cap H_{2}$ whose image $X \in$ $\mathscr{L} \otimes_{\mathscr{O}} \mathfrak{m}_{v}^{2} / \mathfrak{m}_{v}^{3}$ projects to a non-zero element of $\mathscr{M} / \widetilde{\mathscr{M}} \otimes_{\mathscr{O}} \mathfrak{m}_{v}^{2} / \mathfrak{m}_{v}^{3}$. Consider the following commutative diagram, where the vertical arrow on the left hand side is surjective by the inductive assumption:

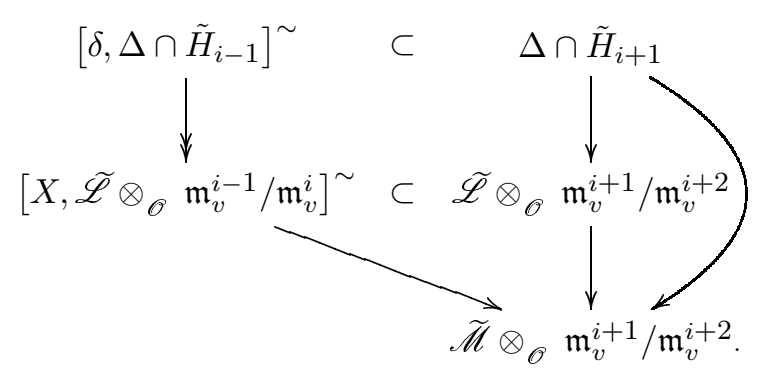

To analyze the oblique map, note first that under our present assumptions the pairing $\widetilde{\mathscr{L}} \times \widetilde{\mathscr{L}} \rightarrow \widetilde{\mathscr{M}}$ vanishes by Proposition 1.2 (a). On the other hand, by Proposition $1.2\left(\right.$ b) we have $[\mathscr{L}, \widetilde{\mathscr{L}}]^{\sim}=\widetilde{\mathscr{L}}$. Recall that $\mathscr{L} / d \pi(\widetilde{\mathscr{L}}) \cong \mathscr{M} / \widetilde{\mathscr{M}}$ has rank one. Thus for any element $Y \in \mathscr{L}$ which maps to a generator of $\mathscr{M} / \widetilde{\mathscr{M}}$, we find that $[Y, \widetilde{\mathscr{L}}]^{\sim}$ maps onto $\widetilde{\mathscr{M}}$. It follows that the oblique map in the above diagram is surjective.

Altogether we can now deduce that the composite vertical map on the right hand side is surjective. Thus Proposition 12.1 (b) implies that the upper vertical map is surjective, as desired.

Lemmas 12.7 and 12.13 imply Proposition 12.4, which in turn implies Proposition 12.3 .

\section{Strong approximation at compact places}

Now we can deduce Theorem 0.2 from the preceding results, using only group theory. The main points are Theorem 5.3 on weak approximation and the following fact. As in Section 5 we let $\Gamma_{V}^{\prime}$ denote the closure of the image of $\Gamma^{\prime}$ in $\tilde{G}\left(F_{V}\right)$. Recall that $S_{\Gamma}$ denotes the set of places where the image of $\Gamma$ does not lie in a compact subgroup.

Proposition 13.1. There exists a finite set of places $V_{0}$, which is disjoint from $S_{\Gamma}$, such that for every finite set of places $V$ that contains $V_{0}$ and is disjoint from $S_{\Gamma}$, 
we have

$$
\Gamma_{V}^{\prime}=\Gamma_{V_{0}}^{\prime} \times \prod_{v \in V \backslash V_{0}} \tilde{\mathscr{G}}\left(\mathscr{O}_{v}\right)
$$

Proof. Let us first clarify some terminology. By a quotient of a topological group we mean the factor group by any closed normal subgroup. Thus two quotients are equal if and only if the respective normal subgroups coincide.

Now fix a finite set of places $S$ which is as large as required by all propositions so far. Since $\Gamma_{S \backslash S_{\Gamma}}^{\prime}$ is a compact subgroup of $\prod_{v \in S \backslash S_{\Gamma}} \tilde{G}\left(F_{v}\right)$, it possesses only finitely many non-abelian finite simple quotients. Let $\Omega_{1}, \ldots, \Omega_{r}$ denote these distinct simple quotients, and let $N$ be the maximum of their orders. Let $V_{0}$ be the union of $S \backslash S_{\Gamma}$ with the set of places $v \notin S$ for which $\left|\mathscr{G}\left(k_{v}\right)^{\text {der }}\right| \leq N$. We will prove the proposition with this choice.

We proceed by induction on $V$. Consider any finite set of places $V \supset V_{0}$ for which the desired equality is proved, and any place $v \notin V \cup S_{\Gamma}$. To prove the equality for $V \cup\{v\}$, we must show

$$
\left.\Gamma_{V \cup\{v\}}^{\prime}=\Gamma_{V}^{\prime} \times \widetilde{\mathscr{G}}_{(} \mathscr{O}_{v}\right)
$$

Identifying $\widetilde{\mathscr{G}}\left(\mathscr{O}_{v}\right)$ with $\prod_{w \in V}\{1\} \times \widetilde{\mathscr{G}}\left(\mathscr{O}_{v}\right)$, it suffices to show that the subgroup

$$
\Delta:=\Gamma_{V \cup\{v\}}^{\prime} \cap \widetilde{\mathscr{G}}\left(\mathscr{O}_{v}\right)
$$

is equal to $\mathscr{G}\left(\mathscr{O}_{v}\right)$. By Proposition 12.3 this reduces to the following lemma.

Lemma 13.2. The image of $\Delta$ in $\mathscr{G}\left(k_{v}\right)$ is $\mathscr{G}\left(k_{v}\right)^{\mathrm{der}}$.

Proof. Consider the commutative diagram

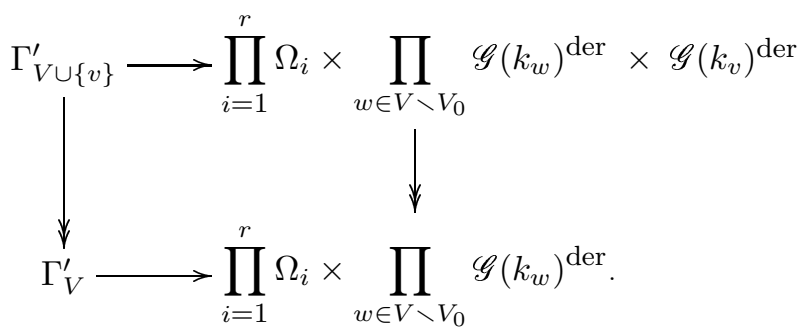

All factors on the right hand side are non-abelian finite simple groups. The inductive assumption implies that the lower homomorphism is surjective. The map $\Gamma_{V \cup\{v\}}^{\prime} \rightarrow \mathscr{G}\left(k_{v}\right)^{\text {der }}$ induced from the upper homomorphism is surjective by Proposition 9.2. Thus if the upper homomorphism is not surjective, by Goursat's lemma its image lies over the graph of an isomorphism between $\mathscr{G}\left(k_{v}\right)^{\text {der }}$ and another 
simple factor. Since $\left|\mathscr{G}\left(k_{v}\right)^{\text {der }}\right|>N \geq\left|\Omega_{i}\right|$ by construction, this factor must be $\mathscr{G}\left(k_{w}\right)^{\text {der }}$ for some $w \in V$. But this is ruled out by Proposition 9.3. Therefore the upper homomorphism is surjective.

As the terms on the lower right hand side are all possible non-abelian finite simple quotients of $\Gamma_{V}^{\prime}$, we deduce that the surjective homomorphism $\Gamma_{V \cup\{v\}}^{\prime} \rightarrow$ $\mathscr{G}\left(k_{v}\right)^{\text {der }}$ does not factor through $\Gamma_{V}^{\prime}$. Thus its restriction to $\Delta$ is non-trivial. Since $\Delta$ is a normal subgroup of $\Gamma_{V \cup\{v\}}^{\prime}$, its image is a normal subgroup of $\mathscr{G}\left(k_{v}\right)^{\text {der }}$. But this group is simple, and the image is non-trivial; hence the image is equal to $\mathscr{G}\left(k_{v}\right)^{\text {der }}$, as desired. This proves the lemma, and thereby finishes the proof of Proposition 13.1.

The ring of adeles of $F$ outside a finite set of places $S$ is the ring

$$
\mathbb{A}_{F}^{S}:=\bigcup_{T}\left(\prod_{v \in T \backslash S} F_{v} \times \prod_{v \notin T} \mathscr{O}_{v}\right)
$$

where $T$ runs through all finite sets of places of $F$ containing those in $S$ and all archimedean ones. The subring associated to any $T$ carries the product topology and is open in $\mathbb{A}_{F}^{S}$. If $S_{i} \subset S$ denotes the subset of places belonging to a simple summand $F_{i}$, we clearly have $\mathbb{A}_{F}^{S}:=\bigoplus_{i=1}^{m} \mathbb{A}_{F_{i}}^{S_{i}}$.

Proof of Theorem 0.2. Taking the limit over all $V$, Proposition 13.1 implies that the closure of $\Gamma^{\prime}$ in $\tilde{G}\left(\mathbb{A}_{F}^{S_{\Gamma}}\right)$ is

$$
\Gamma_{V_{0}}^{\prime} \times \prod_{v \notin V_{0} \cup S_{\Gamma}} \tilde{\mathscr{G}}\left(\mathscr{O}_{v}\right)
$$

On the other hand, by Theorem 5.3 on weak approximation the subgroup

$$
\Gamma_{V_{0}}^{\prime} \subset \prod_{v \in V_{0}} \tilde{G}\left(F_{v}\right)
$$

is already open. Thus the closure of $\Gamma^{\prime}$ in $\tilde{G}\left(\mathbb{A}_{F}^{S_{\Gamma}}\right)$ is open, as desired.

\section{Strong approximation in general}

Finally we prove the equivalence in Theorem 0.3. First assume $0.3(\mathrm{~b})$ and consider any non-empty subset $I \subset\{1, \ldots, m\}$. The closure of $\Gamma^{\prime}$ in $\prod_{i \in I} \tilde{G}_{i}\left(\mathbb{A}_{F_{i}}^{S_{i}}\right)$ is then open. In particular it is not discrete. Since $\pi: \tilde{G} \rightarrow G$ is a finite morphism, it preserves this property, so a fortiori the image of $\Gamma$ in $\prod_{i \in I} G_{i}\left(\mathbb{A}_{F_{i}}^{S_{i}}\right)$ is not discrete. This proves $0.3(\mathrm{a})$, as desired.

Conversely, let us assume 0.3 (a). Consider any finite set of places $V$ which is disjoint from $S$, and let $V=V_{o} \sqcup V_{d}$ be as in Section 6 . 
Lemma 14.1. We have $V_{o}=V$ and $V_{d}=\varnothing$.

Proof. Let $I$ be the set of integers $1 \leq i \leq m$ for which some place of $F_{i}$ is in $V_{d}$. Theorem 6.15 (b) implies that the image of $\Gamma$ in $G\left(F_{V_{d}}\right)$ is discrete. The image of $\Gamma$ in $\prod_{i \in I} G_{i}\left(\mathbb{A}_{F_{i}}^{S_{i}}\right)$ maps isomorphically to this discrete subgroup, so it is itself discrete. Thus the condition 0.3 (a) shows that $I$ is empty. Therefore $V_{d}$ is empty, as desired.

Suppose now that $S_{\Gamma} \backslash S \subset V$. The subset $W_{o}$ of Section 6 is then equal to $V \cap S_{\Gamma}=S_{\Gamma} \backslash S$, and we have $V \backslash W_{o}=V \backslash S_{\Gamma}$. Thus Proposition 6.10 implies

$$
\Gamma_{V}^{\prime}=\tilde{G}\left(F_{S_{\Gamma} \backslash S}\right) \times \Gamma_{V \backslash S_{\Gamma}}^{\prime} .
$$

Taking the limit over all $V$, we deduce that

$$
\text { (closure of } \left.\Gamma^{\prime} \text { in } \tilde{G}\left(\mathbb{A}_{F}^{S}\right)\right)=\tilde{G}\left(F_{S_{\Gamma} \backslash S}\right) \times\left(\text { closure of } \Gamma^{\prime} \text { in } \tilde{G}\left(\mathbb{A}_{F}^{S \cup S_{\Gamma}}\right)\right) \text {. }
$$

On the other hand, Theorem 0.2 implies that the second factor on the right hand side is open in $\tilde{G}\left(\mathbb{A}_{F}^{S \cup S_{\Gamma}}\right)$. Thus the left hand side is open in $\tilde{G}\left(\mathbb{A}_{F}^{S}\right)$. This is just the desired assertion $0.3(\mathrm{~b})$, so Theorem 0.3 is proved.

\section{References}

[1] Artin, E., The orders of the classical simple groups, Comm. Pure Appl. Math. 8 (1955), 455-472.

[2] Borel, A., Linear algebraic groups, GTM 126, Springer-Verlag, New York 1991.

[3] Borel, A., Tits, J., Compléments à l'article: Groupes réductifs, Publ. Math. IHES 41 (1972), 253-276.

[4] Carter, R. W., Simple Groups of Lie Type, Wiley 1972.

[5] Cline, E., Parshall, B., Scott, L., Cohomology of finite groups of Lie type I, Publ. Math. IHES 45 (1975), 169-191.

[6] Cline, E., Parshall, B., Scott, L., Cohomology of finite groups of Lie type II, J. Algebra 45 (1977), 182-198.

[7] Curtis, C. W., Chevalley groups and related topics, in: Finite Simple Groups, Proceedings of an instructional conference, Powell, M. B., Higman, G. (Eds.), Academic Press, 1971, pp. $135-189$.

[8] Demazure, M., Grothendieck, A., (Eds.), Schémas en Groupes I-III, Séminaire de Géométrie Algébrique du Bois Marie 1962/64, SGA3, Lect. Notes Math. 151-153, Springer-Verlag, 1970.

[9] Grothendieck, A., Étude locale des schémas et des morphismes de schémas, Élements de Géométrie Algébrique IV, EGA4, Publ. Math. IHES 20 (1964), 24 (1965), 28 (1966), 32 (1967).

[10] Harder, G., Über die Galoiskohomologie halbeinfacher Matrizengruppen, III, J. Reine Angew. Math. 274/275 (1975), 125-138.

[11] Helgason, S., Differential geometry, Lie groups, and symmetric spaces, Academic Press, 1978.

[12] Hiss, G., Die adjungierten Darstellungen der Chevalley-Gruppen, Arch. Math. 42 (1984), 408-416. 
[13] Hogeweij, G. M. D., Almost Classical Lie Algebras I, Indagationes Math. 44 (1982), 441460.

[14] Kneser, M., Strong approximation, in: Algebraic Groups and Discontinuous Subgroups, Proc. Symp. Pure Math. Vol. 9, AMS, Providence 1966, pp. 187-196.

[15] Larsen, M. J., Pink, R., Finite subgroups of algebraic groups, Preprint (September 1998), 61 p., to appear in $J . A M S$.

[16] Pink, R., Compact subgroups of linear algebraic groups, J. Algebra 206 (1998), 438-504.

[17] Platonov, V., Rapinchuk, A., Algebraic Groups and Number Theory, Academic Press, Boston 1994.

[18] Prasad, G., Strong approximation for semi-simple groups over function fields, Annals of Math. 105 (1977), 553-572.

[19] Prasad, G., Elementary proof of a theorem of Bruhat-Tits-Rousseau and of a theorem of Tits, Bull. Soc. math. France 110 (1982), 197-202.

[20] Steinberg, R., Automorphisms of finite linear groups, Canad. J. Math. 12 (1960), 606-615.

[21] Weisfeiler, B., Strong approximation for Zariski dense subgroups of semi-simple algebraic groups, Annals of Math. 120 (1984), 271-315.

Richard Pink

ETH Zürich

Departement Mathematik

CH-8092 Zürich

Switzerland

e-mail: pink@math.ethz.ch

(Received: August 31, 1999) 